\title{
ACCURATE STELLAR KINEMATICS AT FAINT MAGNITUDES: APPLICATION TO THE BOÖTES I DWARF SPHEROIDAL GALAXY
}

\author{
Sergey E. Koposov ${ }^{1,2}$, G. Gilmore ${ }^{1}$, M. G. Walker ${ }^{1,3}$, V. Belokurov ${ }^{1}$, N. Wyn Evans ${ }^{1}$, M. Fellhauer ${ }^{4}$, W. Gieren ${ }^{4}$, \\ D. Geisler ${ }^{4}$, L. Monaco ${ }^{4,5}$, J. E. Norris ${ }^{6}$, S. OKamoto $^{1}$, J. Peñarrubia ${ }^{1}$, M. Wilkinson $^{7}$, \\ R. F. G. WYSE ${ }^{8}$, AND D. B. ZUCKER ${ }^{9,10}$ \\ ${ }^{1}$ Institute of Astronomy, Madingley Road, Cambridge CB3 0HA, UK \\ ${ }^{2}$ Sternberg Astronomical Institute, Universitetskiy pr. 13, 119992 Moscow, Russia \\ ${ }^{3}$ Harvard-Smithsonian Center for Astrophysics, Cambridge, MA 02138, USA \\ ${ }^{4}$ Departamento de Astronomia, Universidad de Concepcion, Chile \\ ${ }^{5}$ European Southern Observatory, Alonso de Cordova 3107, Casilla 19001, Santiago 19, Chile \\ ${ }^{6}$ Research School of Astronomy \& Astrophysics, The Australian National University, Mount Stromlo Observatory, \\ Cotter Road, Weston, ACT 2611, Australia \\ ${ }^{7}$ Department of Physics and Astronomy, University of Leicester, Leics, UK \\ 8 Department of Physics \& Astronomy, The Johns Hopkins University, 3900 N. Charles Street, Baltimore, MD 21218, USA \\ ${ }^{9}$ Department of Physics and Astronomy, Macquarie University, North Ryde, NSW 2109, Australia \\ ${ }^{10}$ Australian Astronomical Observatory, P.O. Box 296, Epping, NSW 1710, Australia \\ Received 2011 January 4; accepted 2011 May 13; published 2011 July 19
}

\begin{abstract}
We develop, implement, and characterize an enhanced data reduction approach which delivers precise, accurate, radial velocities from moderate resolution spectroscopy with the fiber-fed VLT/FLAMES+GIRAFFE facility. This facility, with appropriate care, delivers radial velocities adequate to resolve the intrinsic velocity dispersions of the very faint dwarf spheroidal $(\mathrm{dSph})$ galaxies. Importantly, repeated measurements let us reliably calibrate our individual velocity errors $\left(0.2 \mathrm{kms}^{-1} \leqslant \delta_{V} \leqslant 5 \mathrm{~km} \mathrm{~s}^{-1}\right)$ and directly detect stars with variable radial velocities. We show, by application to the Boötes I dSph, that the intrinsic velocity dispersion of this system is significantly below $6.5 \mathrm{~km} \mathrm{~s}^{-1}$ reported by previous studies. Our data favor a two-population model of Boötes I, consisting of a majority "cold" stellar component, with velocity dispersion $2.4_{-0.5}^{+0.9} \mathrm{~km} \mathrm{~s}^{-1}$, and a minority "hot" stellar component, with velocity dispersion $\sim 9 \mathrm{~km} \mathrm{~s}^{-1}$, although we cannot completely rule out a single component distribution with velocity dispersion $4.6_{-0.6}^{0.8} \mathrm{~km} \mathrm{~s}^{-1}$. We speculate that this complex velocity distribution actually reflects the distribution of velocity anisotropy in Boötes I, which is a measure of its formation processes.
\end{abstract}

Key words: galaxies: dwarf - galaxies: individual (Boötes I) - galaxies: kinematics and dynamics - methods: data analysis - techniques: radial velocities

Online-only material: color figures

\section{INTRODUCTION}

There is a continuing interest in analysis of the number, nature, masses, and evolutionary histories of the dwarf spheroidal (dSph) satellite galaxies, found in moderate numbers around both the Milky Way Galaxy and M31 in the Local Group. They have typical half-light radii greater than $100 \mathrm{pc}$, low surface brightnesses $\left(\sim 25-30 \mathrm{mag} \operatorname{arcsec}^{-2}\right.$ ), and central velocity dispersions of several $\mathrm{km} \mathrm{s}^{-1}$, implying that the luminous component is embedded in a dominant extended dark matter halo $\left(M / L_{V} \sim 10-100 M / L_{V, \odot}\right.$; Mateo 1998; Gilmore et al. 2007). Their chemical abundances are low in the mean, correlate with $\mathrm{dSph}$ system luminosity, show real intrinsic dispersion, and have chemical element ratios systematically different from those of Galactic halo field stars over the metallicity range covering most Galactic halo stars (Kirby et al. 2009; Norris et al. 2010a; Tolstoy et al. 2009). Their stellar populations differ systematically from those in the Galactic field. All the astrophysical evidence shows that they are the oldest surviving bound systems, probably forming very early from purely primordial gas, e.g., Norris et al. (2010a). How do they relate to the very large numbers of surviving dark matter halos predicted by standard structure formation models?

The most luminous dSphs around the Milky Way, the "classical" dSphs, were discovered photometrically through the second half of the twentieth century, apart from the nearest and largest, the Sgr dSph, which was discovered in positionvelocity-photometry phase space (Ibata et al. 1994, 1995). More recently, following the availability of the large area photometric data from the Sloan Digital Sky Survey (SDSS; York et al. 2000), a three-times larger sample of dSph has been discovered, primarily of dSphs with lower intrinsic luminosities, extending to the "ultrafaint" dSphs (Willman et al. 2005; Belokurov et al. 2006, 2007, 2008, 2009; Zucker et al. 2006a, 2006b; Walsh et al. 2007). These objects have extremely low surface brightnesses (down to $30 \mathrm{mag} \operatorname{arcsec}^{-2}$ ) and low luminosities (down to $\left.L_{V} \sim 10^{3} L_{V, \odot}\right)$, such that the presence of a single giant star can substantially affect the luminosity of the entire galaxy (Martin et al. 2008).

Knowledge of the numbers and masses of dwarf satellites remains crucial for understanding local galaxy formation, since high-resolution cosmological $N$-body simulations of galaxy formation generally predict a number of dark matter "subhaloes" that is still an order of magnitude larger than the number counted in and extrapolated from observations (the "Missing Satellites Problem," Klypin et al. 1999; Moore et al. 1999). Considerable efforts have been expended in simulating dwarf galaxy formation (Barkana \& Loeb 1999; Somerville 2002; Benson et al. 2003; Ricotti 2010), attempting to lessen the tension between predictions from simulations and the observations (Koposov et al. 2009; Macciò et al. 2010; Cooper et al. 2010). Modeling the census of $\sim 25$ known Milky Way 
dSphs for survey incompleteness (Koposov et al. 2008; Walsh et al. 2009), one can estimate that the number of such galaxies within the halo of the Milky Way could be several hundred, although with a significant error bar (Tollerud et al. 2008; Koposov et al. 2009). Nearly $30 \mathrm{dSph}$ are now known around M31, where the whole area has been studied, but not to such low luminosities. Empirical constraints on the dark halo masses of these objects are required in order to say whether these discoveries represent a step toward consistency with standard cosmological models.

The structure of the paper is as follows: We first outline our specific motivation, deriving reliable velocity dispersions in faint dSph galaxies. We then introduce the Boötes I galaxy and our observational approach, which is designed to test the reliability with which we can derive accurate radial velocities of faint stars. In Section 4, we describe our data reduction procedures, which are developed to ensure use of the full information content in the raw data. In Section 5, we describe our enhanced procedure for fitting the stellar spectra and deriving precise, accurate radial velocities as well as our approach to understanding the errors. In Section 6, we quantify how we detect radial velocity variations. Section 7 compares our results with available literature studies. In Section 8 , we describe our statistical methodology to determine velocity dispersions from kinematic data, and apply this to the Boötes I dwarf galaxy. In Section 9, we conclude our study with some discussion of the astrophysical implications.

\section{MEASURING VELOCITY DISPERSIONS IN dSph GALAXIES}

Several factors complicate determinations of dSph galaxy masses. The most basic stems from the fact that we can measure only line-of-sight velocities of dSph stars. As a result, any estimation of a dSph's total mass must contend with the fundamental degeneracy between mass and anisotropy in the velocity distribution (e.g., Wilkinson et al. 2002; Kleyna et al. 2002). The second basic constraint, one that is more general, is that we very rarely see an outer declining dispersion profile (or rotation curve), allowing determination of a "total" mass. A third key limit comes from having to suppose that the dSph can be considered in dynamic equilibrium - an assumption whose validity is certainly not obvious for those extreme systems currently within a few tens of $\mathrm{kpc}$ of the Galactic center. Accepting the equilibrium assumption, and using just radial velocities, several recent studies showed that the most robust mass that it is possible to estimate for faint dwarfs is the mass enclosed within the half-light radius (Penarrubia et al. 2008; Walker et al. 2009; Wolf et al. 2010). However, estimates of even this quantity meet with observational hurdles: one is that the ultrafaint dwarf galaxies have a very small number of stars $\left(\sim 10^{2}-10^{4}\right)$, so for some objects the number of targets available for spectroscopic observations with even the largest telescopes is limited to a few tens (Simon \& Geha 2007) of predominantly faint stars, for which radial velocity errors may be of the order of or larger than the velocity dispersion of the system. Another difficulty of measuring the small velocity dispersions for the faint dwarf galaxies using small numbers of stars is that it is still unclear how much they can be affected by the binary stars which must be in the sample (Olszewski et al. 1996; Hargreaves et al. 1996; Minor et al. 2010; McConnachie \& Côte 2010). A perennial limitation in all these studies is data quality-are systematic and random uncertainties in the data quantified as precisely as is claimed?
We have two ambitions in this study. The first is to improve our knowledge of the Boötes I dSph galaxy. Its high scientific interest is introduced in the first subsection below. Our second aim is to improve our knowledge of how reliably and accurately we can measure radial velocities of $\mathrm{dSph}$ member stars. Our observations have been designed to allow us to derive radial velocities with sufficient precision, and with sufficiently wellknown accuracy, to resolve the intrinsic dispersion in a typical faint dSph galaxy, to properly understand our measurement errors, to quantify the fraction of objects with variable radial velocities, and hence to measure the intrinsic velocity dispersion with reliable precision. This study provides a template for how reliably, and how accurately, velocity dispersions can really be determined in the faintest $\mathrm{dSph}$ galaxies. These ultrafaint $\mathrm{dSphs}$ have very few apparently bright member stars, so one is forced to determine precision velocities for very faint stars from moderate spectral resolution and moderate signal-to-noise ratio $(\mathrm{S} / \mathrm{N})$ spectra, with the relevant velocity accuracy being at most a few percent of 1 pixel in the observed spectrum. The stars additionally tend to be very metal-poor, weakening absorption line strengths.

Kinematic studies of ultrafaint $\mathrm{dSphs}$ are especially challenging due to the fact that the measured velocity dispersions, the typical errors of individual velocities, and the expected contribution of binary stars are all similar, at a few $\mathrm{km} \mathrm{s}^{-1}$. The previously studied SDSS dSphs have reported velocity dispersions as small as $\sim 3 \mathrm{~km} \mathrm{~s}^{-1}$ (Simon \& Geha 2007; Martin et al. 2007). McConnachie \& Côte (2010) demonstrate that for such cold dispersions, it is difficult to disentangle contributions from random (i.e., reflective of the underlying gravitational potential) and binary-orbital motions. It may even be that some of the apparently small extreme ultrafaint systems are not (currently) dark matter dominated, but are dissolved star clusters or tidal debris. In such a case the velocity dispersion could be substantially lower than $\sim 3 \mathrm{~km} \mathrm{~s}^{-1}$. Could we detect such an absence of dark matter?

In this situation it is critical to accurately account for velocity errors-when propagated through calculations, optimistic/ pessimistic errors result in over/underestimates of the true velocity dispersions. That is, how important are the uncertainties on the error bars? We explore the severity of this risk with some very simple Monte Carlo simulations, in which we draw velocity samples of 100 stars (slightly larger than our present Boötes I sample), in order to minimize sampling error and thereby to isolate bias due to inadequate resolution) from a Gaussian distribution with true dispersion $\sigma_{\text {true }}$, and then add to each velocity a "true" error drawn from a second Gaussian with dispersion $\delta_{\text {true }}$. In real observations the true error is unknown; we therefore measure the velocity dispersion of each artificial sample after adopting velocity errors $k \delta_{\text {true }}$. The factor $k$ is held constant for a given sample and represents the accuracy of the adopted errors: $k=1$ if the adopted errors are accurate; $k>1$ for unduly pessimistic errors; $k<1$ for overoptimistic errors. We repeat the simulation for values $k=0.5,0.75,1.0,1.25,1.5$, and $\delta_{\text {true }} / \sigma_{\text {true }}=0.1,0.2, \ldots, 1.5$; the latter sequence lets us examine accuracy as the true velocity dispersion dominates, or is dominated by, the measurement errors.

Figure 1 displays the squared ratio of the measured to the true velocity dispersion, $\sigma_{\text {measured }}^{2} / \sigma_{\text {true }}^{2}$, as a function of $\delta_{\text {true }} / \sigma_{\text {true }}$. Plotted points represent average values from $10^{4}$ trials at each accuracy level. We recover the required outcome: that if measurement errors are known perfectly, intrinsic dispersions can be measured accurately, even if they are dominated by 


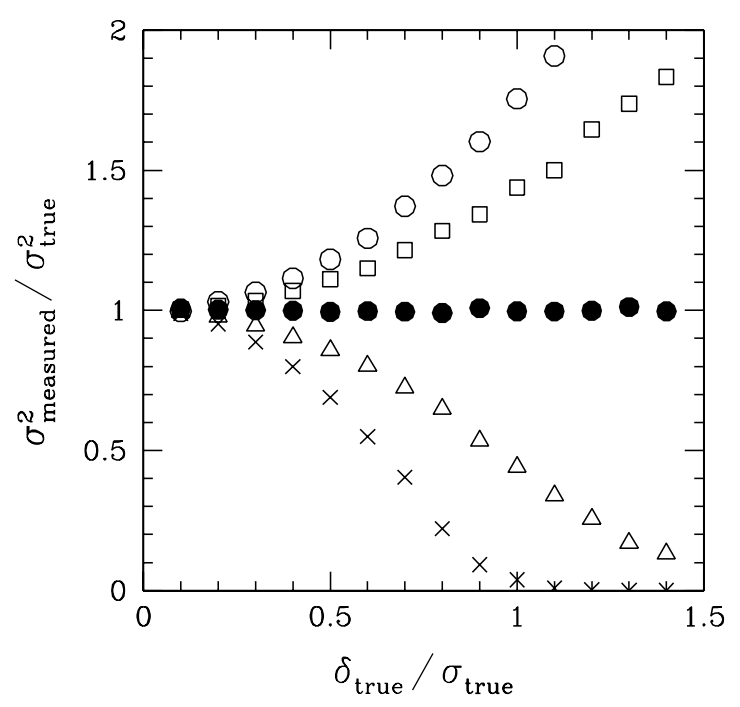

Figure 1. Accuracy of simulated velocity dispersion estimates as a function of the ratio of measurement errors to the true velocity dispersion. Filled circles represent cases in which the measurement errors are known perfectly. Open circles and open squares represent cases in which the errors used in the analysis are underestimated (optimistic) by a factor of 0.5 and 0.75 , respectively. Open triangles and crosses represent cases in which the adopted errors are overestimated (pessimistic) by a factor of 1.25 and 1.5 , respectively.

measurement errors. However, as the velocity error becomes a significant fraction of the true dispersion, deductions quickly become unreliable for even modestly misjudged errors. There is a particular danger of grossly underestimating velocity dispersions with pessimistic errors: when $\delta_{\text {true }} \sim \sigma_{\text {true }}$, the measured dispersion is only $65 \%(20 \%)$ of the true value if the errors are overestimated by a factor of $k=1.25(k=1.5)$.

These results emphasize the caution necessary when measuring and interpreting small velocity dispersions. Two prerequisites for obtaining reliable results are (1) sufficient resolution such that typical velocity errors are smaller than the velocity dispersion, and (2) accurate estimates of the velocity errors. We designed this study with that lesson in mind.

\section{THE BOÖTES I DWARF GALAXY}

Boötes I was one of the first new dSphs discovered using the SDSS photometric survey, by Belokurov et al. (2006). Boötes I has a number of interesting properties, but seems representative of the group of newly discovered intrinsically faint $\mathrm{dSph}$ galaxies which are (reasonably) far from the Galactic center. Many recent studies are available. Photometric and stellar population studies have been completed by Hughes et al. (2008), de Jong et al. (2008), Okamoto (2010); RR Lyrae variability studies by Siegel (2006), Dall'Ora et al. (2006); spectroscopic abundance studies by Muñoz et al. (2006), Martin et al. (2007), Norris et al. (2008, 2010b, 2010a); and an H I $21 \mathrm{~cm}$ search by Bailyn \& Ford (2007). Of specific relevance here, two kinematic studies are available. Muñoz et al. (2006) obtained spectra of red giant branch (RGB) candidates over part of Boötes I, selected from SDSS DR4. They used the WIYN telescope and the Hydra multifiber spectrograph. Their data (for a seven-member-star sub-sample) yielded a systemic velocity of $95.6 \pm 3.4 \mathrm{~km} \mathrm{~s}^{-1}$, and a central velocity dispersion of $6.6 \pm 2.3 \mathrm{~km} \mathrm{~s}^{-1}$. They derived $\sigma_{0}=9.0 \pm 2.2 \mathrm{~km} \mathrm{~s}^{-1}$ from a 12-member-star sample, a value that was adopted in a later analysis by Wolf et al. (2010). Martin et al. (2007) observed candidate Boötes I red giants from SDSS (DR4) with Keck/DEIMOS, finding a mean velocity of $99.9 \pm 2.4 \mathrm{~km} \mathrm{~s}^{-1}$, with central velocity dispersion $\sigma=6.5_{-1.3}^{+2.1} \mathrm{~km} \mathrm{~s}^{-1}$ for their final sample of 24 member stars with small $\left(\delta_{v} \lesssim 10 \mathrm{~km} \mathrm{~s}^{-1}\right)$ velocity uncertainties. From their kinematics, Muñoz et al. (2006) deduced a mass of $1.1_{-0.5}^{+1.3} \times 10^{7} M_{\odot}$. Wolf et al. (2010) adopt the high-velocity dispersion of $9.0 \pm 2.2 \mathrm{~km} \mathrm{~s}^{-1}$, by accepting the superset of the data of Muñoz et al. (2006). From this they deduce a correspondingly higher mass.

In summary, Boötes $\mathrm{I}$ is some $65 \mathrm{kpc}(m-M=19.07)$ distant, has absolute luminosity $M_{V, \odot}=-5.9$, is devoid of $\mathrm{H} \mathrm{I}$, has somewhat elliptical (ellipticity $=0.2$ ) morphology, has a half-light radius of $240 \mathrm{pc}$, has an apparently exclusively old metal-poor stellar population, with a significant blue straggler sub-population, has a mean $[\mathrm{Fe} / \mathrm{H}]$ metallicity of -2.55 , has an intrinsic abundance dispersion with formal $\sigma=0.45$, with a range in $[\mathrm{Fe} / \mathrm{H}]$ of at least $1.7 \mathrm{dex}$, and has at least one member star which has $[\mathrm{Fe} / \mathrm{H}]=-3.7$.

Boötes I is ideal for a more detailed kinematic study. Observationally, Boötes I has kinematic data available from two quite different spectrographs (one fiber-coupled, one slit), so that both an internal and an external test of the accuracy of our data is possible. It is at intermediate distance from the Galactic center ( $70 \mathrm{kpc}$ Galactocentric), far enough that tidal effects need not be dominant, yet close enough that observations far enough down the giant branch to obtain useful statistical samples are feasible, in spite of its (interestingly) low intrinsic luminosity. It has extremely low surface density, so it is a clear test case for galaxy formation models, none of which naturally create very large, very low surface brightness galaxies. It has extremely low metallicity stars. The abundance results suggest that Boötes I is a survivor of a true primordial system, quite likely forming prior to reionization, enhancing its interest. Its velocity dispersion is reported as being at least $6 \mathrm{~km} \mathrm{~s}^{-1}$, a value that is feasible to measure to high accuracy. It seems to have an extremely high apparent mass-to-light ratio, again enhancing its intrinsic interest. It is visible from the Very Large Telescope (VLT). The primary challenge is that it is large on the sky. Our present study, involving a single FLAMES field, as with those in the literature, covers only the central half-light radius of Boötes I.

\subsection{Target Selection}

For these observations, we selected target stars with a sufficiently wide range of apparent magnitude that a single observation of the brightest stars would provide similar $\mathrm{S} / \mathrm{N}$ to the final $\mathrm{S} / \mathrm{N}$ of the fainter stars, integrated over 16 identical single exposures. Thus, by comparing the actual repeatability of velocities for the brighter stars, we could verify if the deduced accuracy calculated for the faintest stars was reliable. Additionally, by summing the brighter star exposures, we could readily test for any systematic floor in delivered accuracy at some high signal-to-noise value. We were fortunate to have access to the VLT FLAMES spectrograph, which, being fiber-fed, substantially reduces the extra complexity of slit-centering errors, which unavoidably plagues slit spectroscopy (Hargreaves et al. 1994).

We used the FLAMES spectrograph at the $8.2 \mathrm{~m}$ Kueyen (VLT/UT2) telescope at Cerro Paranal, Chile, to acquire spectra of individual stellar targets in Boötes I. Observations took place in service mode during 2009 February to March in fulfillment of ESO Programme 182.B-0372A (PI: Gilmore). For this program we used FLAMES in UVES-Fiber mode (Pasquini et al. 2002); that is, 130 fibers fed the mediumresolution Giraffe spectrograph while eight additional fibers fed 

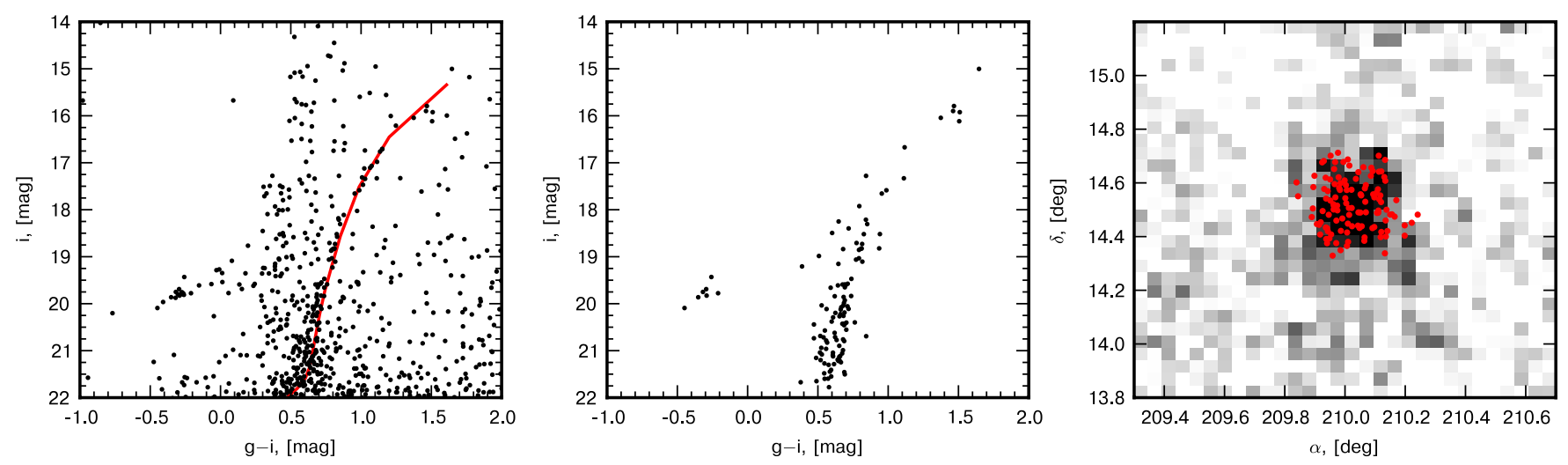

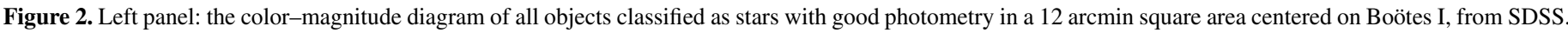

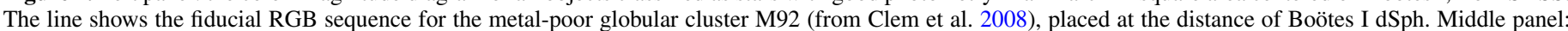

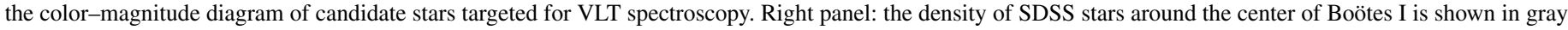
scale, the targeted stars are shown by red points. For comparison, the half-light radius of Boötes I is 12.5 arcmin.

(A color version of this figure is available in the online journal.)

the high-resolution UVES spectrograph. The Giraffe spectra sample the region 8180-9375 $\AA$, in LR08 setup, including the prominent Ca II-triplet (CaT) absorption feature, with resolving power $R \sim 6500$. The UVES spectra results will be described elsewhere (L. Monaco \& G. Gilmore 2011, in preparation). Here, we present results based primarily on the Giraffe spectra.

In order to maximize the probability of observing Boötes I members, we selected stellar targets based on colors and magnitudes of the stars. The left-hand panel of Figure 2 displays the color-magnitude diagram (CMD) of all stars (including foreground) within 12 arcmin of the center of Boötes I, from SDSS photometry and with magnitudes corrected for extinction using the dust maps of Schlegel et al. (1998). Recall that the halflight radius of Boötes I is greater than 12 arcmin - we are sampling only the very inner regions in this (first) study. Boötes I's low luminosity presents a challenge to spectroscopic studies, as the paucity of bright RGB candidates limits sample size. As the RGB of Boötes I together with a few blue horizontal branch (BHB) stars are clearly visible on the left panel of Figure 2 (see also Figure 1 from Belokurov et al. 2006), the targets were selected using a CMD mask covering the location of RGB and BHB stars. The middle panel of Figure 2 plots the CMD for our FLAMES targets, which include stars up to 2 mag fainter than the horizontal branch. The UVES targets are all brighter than $r \sim 18$, while the limiting magnitude of $r \sim 22$ for our Giraffe targets represents a compromise between quantity and quality of the spectra in our sample. As noted above, we retain several (relatively) bright stars of low a priori membership probability as a key part of our internal quality checks.

The second compromise involved the trade-off between target numbers and adequate data to test our delivered velocity accuracy. In order to obtain useful spectra for the faintest targets and to implement our test of achievable precision, we observe only a single field with a single target configuration centered on Boötes I, building S/Ns by repeated science exposures. (The alternative would have been to reallocate those fibers allocated to brighter target stars after one or a few integrations, to increase observed numbers.) Our adopted strategy is well suited for service mode observations, given the excellent stability of the FLAMES instrumentation. Over the six weeks of our observing program, we obtained 16 individual science exposures, typically of 45 minutes each. The total exposure time on our field was $11.5 \mathrm{hr}$.

\section{DATA REDUCTION}

We first summarize the sequence of our data processing before providing a detailed description below.

1. Default basic processing using the ESO pipeline (bias subtraction, flat fielding, wavelength calibration, extraction).

2. Wavelength recalibration of each extracted spectrum using sky emission lines.

3. Combining sky fibers for the determination of the master sky spectrum for each frame and subtracting from individual extracted objects.

4. Combining the individual frames into the co-added spectra. Co-added spectra are used then to reject cosmic rays from individual spectra.

5. Spectral fitting of the co-added spectra. We determine the best-fit template and approximate velocity.

6. Spectral fits of individual (not co-added) spectra using the best-fit template.

The initial data processing was done using the giraf-3.8.1 pipeline provided by ESO with some modifications and bug fixes described below. The important bug we discovered and fixed in the pipeline was related to the computation of the variance spectra, which had been incorrectly scaled by the pipeline. Another modification that we applied to the pipeline was related to the extracted but not rebinned spectra. Our goal was to minimize the number of rebinning operations on the data, so we needed to produce non-rebinned-extracted spectra as well as the corresponding wavelength solutions. As the original ESO pipeline does not provide these, we modified our version of the pipeline to output the necessary information, in un-rebinned pixel space. All the further discussion of the data reduction will take into account that each individual spectrum is in its native pixel space, and so each spectrum is on a different wavelength grid. By contrast, the common approach has all the data rebinned to a common wavelength scale, and often a common dispersion, prior to all subsequent analyses.

\subsection{Wavelength Calibration}

The wavelength calibration was done using the standard thorium-argon arc spectra, which were taken during the day, not in parallel with the nighttime observations. According to the GIRAFFE user manual this calibration will deliver a 


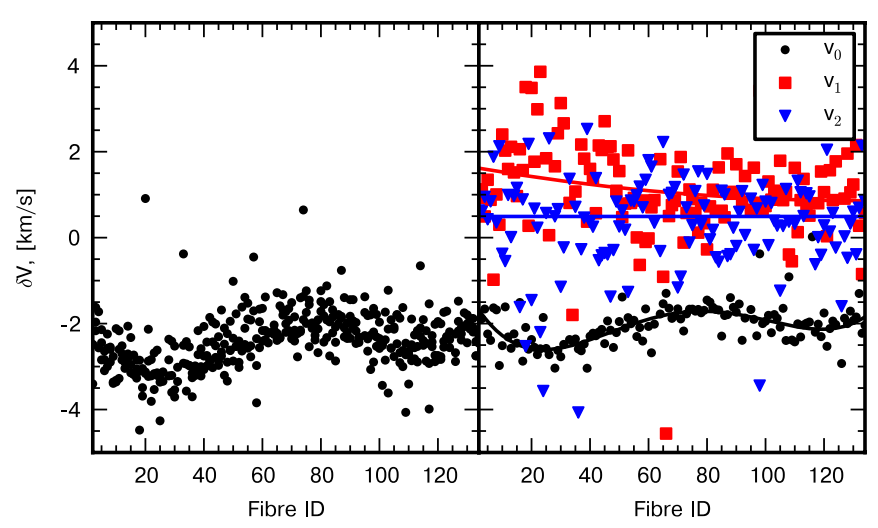

Figure 3. Left panel: velocity offsets from the ThAr arc wavelength fit, determined using the three skylines 8310.7246, 8415.2422, 8452.2656 plotted for a single data frame, as a function of fiber ID number. Right panel: the parameters of the polynomial wavelength corrections $v_{0}, v_{1}, v_{2}$ from Equation (1) as a function of fiber ID. Different symbols show the measurements for individual fibers, while the lines show the polynomial fits to these measurements. Further explanation is in the text.

(A color version of this figure is available in the online journal.)

precision limited only by spectral resolution and $\mathrm{S} / \mathrm{N}$, with a delivered instrumental floor at very high spectral resolution of $30 \mathrm{~m} \mathrm{~s}^{-1}$. After analyzing the extracted spectra, we noticed that the skylines had systematic velocity shifts of the order of $1-3 \mathrm{~km} \mathrm{~s}^{-1}$. The left panel of Figure 3 shows these offsets measured using three isolated skylines $(8310.7246 \AA$, 8415.2422 $\AA$, and 8452.2656 $\AA$ ) as a function of fiber ID for one of the GIRAFFE frames. A waving pattern of velocity offsets versus the fiber IDs is clearly visible. In order to correct for these velocity offsets in each fiber we used all (around 100) bright skylines simultaneously. For each fiber we first subtracted the continuum from the sky or from the stellar spectra, then we fitted the residual spectrum in pixel space by a sum of $n \times$ LSFs (line spread functions) at the locations given by the catalog of skylines from Hanuschik (2003) after applying the polynomial correction to the wavelengths:

$$
\lambda=\tilde{\lambda}\left(1+\frac{1}{c}\left(v_{0}+v_{1} \frac{\tilde{\lambda}-8400}{500}+v_{2}\left(\frac{\tilde{\lambda}-8400}{500}\right)^{2}\right)\right)
$$

Although the number of lines is high and the number of parameters is high also, the fit may be done easily via sparse matrix operations. As a result of this procedure we derive the best polynomial correction to the spectra $v_{0}, v_{1}, v_{2}$. The results of determination of $v_{0}, v_{1}, v_{2}$ for all the fibers on one particular GIRAFFE frame are shown in the right panel of Figure 3. Despite considerable noise in the measured coefficients from fiber to fiber, overall the coefficients vary smoothly with fiber ID (fiber ID is enumerating fibers along the slit and on the CCD across the dispersion). In order to further reduce the fiberto-fiber noise in the wavelength corrections, we fitted the $v_{0}, v_{1}, v_{2}$ coefficients by a set of low-order polynomials (fifth order for $v_{0}$, second order for $v_{1}$, and zeroth order for $v_{2}$ ). The results of the fit are shown as colored lines in the right panel of Figure 3. We use those curves to determine the values of the parameters $v_{0}, v_{1}, v_{2}$ for each fiber, and then substitute those values into Equation (1) in order to determine the final wavelength solution for each fiber.

\subsection{Sky Subtraction}

In each Giraffe frame we allocated 12 target fibers to sky, with sky areas chosen to have no object detected by SDSS nearby. In order to obtain an average sky spectrum for each frame, we first applied the corrections from Section 4.1, then rebinned the sky spectra, with two-fold oversampling, from each individual fiber to the common wavelength grid. We then multiplied each of the individual sky spectra by a low-order (2) polynomial of wavelength to ensure that all rebinned sky spectra had the same photon-count normalization with wavelength, and median-combined the rebinned sky spectra, creating an "average" sky spectrum. For the error spectrum of the combined sky spectrum, we used the median absolute deviation (MAD), scaled by $1.4826 \sqrt{\frac{\pi}{2}} /$ sqrt(number of sky spectra), following Rousseeuw \& Croux (1993).

The average sky was then subtracted from each individual fiber on the frame. In order to perform this subtraction, we had to rebin the average sky spectrum back onto the original pixel wavelength grid of each fiber. After subtracting the scaled sky spectrum from each fiber we also added the variance of the mean sky spectrum to the variance spectrum of each fiber to take into account the uncertainty of the sky determination.

\subsection{Combining Spectra}

Since each object in our sample was observed from 9 to 18 times, we need to co-add the individual spectra. Although we did not use the co-added spectra for the radial velocity determinations, we needed the co-added spectra for certain specific tasks, for example, cosmic-ray rejection in individual exposures.

In order to combine repeated science spectra for a given star, we first made a zero-point wavelength correction to correct for the varying radial velocity component due to Earth's motion, then rebinned each individual sky-subtracted spectrum to a common wavelength grid, as previously done for raw sky spectra. Then we followed a procedure similar to that which we used in the determination of the average sky spectrum. Since our spectra were not flux corrected, before combining the individual spectra we used second-order polynomials to multiply all individual spectra, to ensure that each had the same continuum level across the wavelength range. Then we mediancombined these spectra. For variance spectra we used, as before, the MAD, scaled by $1.4826 \sqrt{\frac{\pi}{2}} / \operatorname{sqrt}$ (number of observations).

Having the co-added spectra for each object allows us to identify bad pixels/cosmic rays/outliers in each individual (not co-added) spectrum. In order to do that, we interpolated the co-added spectra to the wavelength grid of each individual observation, and then we masked out those pixels which were more than $4 \sigma$ above or $6 \sigma$ below the median spectrum. Strictly speaking, that procedure may introduce biases if the spectrum is highly variable from exposure to exposure-in those cases the variable lines may be masked out, but we visually checked all the spectra and did not see any inappropriately masked spectral features.

\section{VELOCITY DETERMINATION AND SPECTRAL FITTING}

The standard approach used for the measurement of stellar velocities (Hargreaves et al. 1994; Koch et al. 2007, 2009; Simon \& Geha 2007; Walker et al. 2007; Baumgardt et al. 2009; Geha et al. 2009; Leaman et al. 2009) has been to crosscorrelate against a template spectrum (Simkin 1974; Tonry \& 
Davis 1979). Although simple and computationally fast, crosscorrelation is known to not perform optimally (Rix \& White 1992; Cappellari \& Emsellem 2004). In fact direct pixel-fitting methods have been widely employed for more than a decade in spectroscopic studies of unresolved stellar populations (Rix \& White 1992; Cappellari \& Emsellem 2004; Chilingarian et al. 2007; Koleva et al. 2009). Methods based on direct pixel fitting provide more realistic error bars and give a better way to treat multiple templates and continuum levels.

In this section, we briefly describe the pixel-fitting method we use, which is similar to the ones described in Rix \& White (1992), Cappellari \& Emsellem (2004), Chilingarian et al. (2007), and Koleva et al. (2009), but with a few differences.

\subsection{Stellar Library}

An important ingredient for direct pixel-fitting methods is the library of template spectra. We decided to use the library of synthetic spectra provided by Munari et al. (2005). The spectra in the library cover a large range of stellar parameters $-2.5<[\mathrm{Fe} / \mathrm{H}]<0.5 ;[\alpha / \mathrm{Fe}]=0,0.4 ; 3000<T_{\text {eff }} / 1 \mathrm{~K}<$ 80,$000 ; 1.5<\log (g)<5 ; 0<V_{\text {rot }} / 1 \mathrm{~km} \mathrm{~s}^{-1}<150$. The highest resolution spectra available from the Munari library are $R \sim 20,000$, which is higher than the resolution of our data $(R \sim 6500)$, so the templates can be easily downsampled to our resolution. While synthetic spectral libraries have obvious limitations and we do not expect a perfect match to the observed stars, the range of stellar parameters covered in the library is large and is significantly better than what we can achieve with stellar libraries of high-resolution spectra for real stars such as ELODIE (Prugniel et al. 2007); see also Kirby et al. (2008). This is particularly true given the extremely low metallicities of the Boötes I member stars (Norris et al. 2010a).

\subsection{Fitting Synthetic Templates to Real Spectra}

Having the stellar template library we construct the model of each observed spectrum as

$$
\operatorname{Model}\left(\lambda, i, v,\left\{p_{j}\right\}\right)=P(\lambda) \cdot T_{i}\left(\lambda\left(1+\frac{v}{c}\right)\right)
$$

where $T_{i}(\lambda)$ is the $i$ th template spectrum from the template grid convolved with the appropriate line spread function $\operatorname{LSF}(\lambda), v$ is the radial velocity of the object, and $P(\lambda)=\sum_{j=0}^{N-1} p_{j} \cdot \lambda^{j}$ is the normalizing polynomial of low degree, $(N-1)$, which takes into account the lack of flux calibration of our spectra as well as any imprecision in our location of the continuum of the template spectra.

Having the spectral model one can then compute the $\chi^{2}$ value by summing scaled residuals over pixels:

$$
\chi^{2}\left(i, v,\left\{p_{j}\right\}\right)=\sum_{k}\left(\frac{S p_{k}-\operatorname{Model}\left(\lambda_{k}, i, v,\left\{p_{j}\right\}\right)}{E S p_{k}}\right)^{2},
$$

where $S p_{k}$ and $E S p_{k}$ are the observed spectra and variance spectra, respectively, and the $\lambda_{k}$ are the wavelengths of the pixels of the extracted spectra. $\operatorname{Model}\left(\lambda_{k}, \ldots\right)$ is the evaluation of the synthetic stellar model spectrum at the wavelengths of the individual pixels. It is important that the original observed spectrum is neither rebinned nor interpolated in any way, as this would lead to correlated noise and information loss.
Equation (3) defines the $\chi^{2}$ or log-likelihood of our model. In order to find the estimated radial velocity and the bestmatched template, we need to minimize the $\chi^{2}$ with respect to all relevant parameters. In fact we are not interested in values of the coefficients of the normalizing polynomials $P(\lambda)$, so we can marginalize over them and derive the joint probability distribution of just the template identification $i$, and the radial velocity, $v$. With a simple analytical computation we can derive that probability distribution:

$$
\operatorname{Prob}(i, v)=(\operatorname{det}(M))^{-\frac{1}{2}} \exp \left(-\frac{1}{2} \mathbf{Y}^{T} M \mathbf{Y}\right)
$$

where $\mathbf{Y}$ is the vector having length $N$, such that

$$
\mathbf{Y}[j](i, v)=\sum_{k} \frac{S p_{k} T_{i}\left(\lambda_{k}\left(1+\frac{v}{c}\right)\right)}{E S p_{k}} \lambda_{k}^{j}
$$

and $M$ is a symmetric square $N \times N$ matrix, such that

$$
M\left[j_{1}, j_{2}\right](i, v)=\sum_{k} \frac{S p_{k}^{2} T_{i}^{2}\left(\lambda_{k}\left(1+\frac{v}{c}\right)\right)}{E S p_{k}^{2}} \lambda_{k}^{j_{1}+j_{2}}
$$

Equation (4) describes the joint probability distribution of an identified template and an associated target star radial velocity $\operatorname{Prob}(i, v)$. In practice, we evaluate this probability for a grid of plausible radial velocities $|v|<500 \mathrm{~km} \mathrm{~s}^{-1}$ (this is effectively a uniform prior on radial velocity) and for our grid of templates (also assuming a uniform prior). This two-dimensional (2D) probability distribution can then be marginalized over template or velocity. The marginalization over template can be used to determine the maximum a posteriori (MAP) estimate of the velocity, $\left(\arg \max _{v} \operatorname{Prob}(v)\right)$, and of the velocity error. The other marginalization identifies the best-fitting template spectrum, and therefore an estimate of stellar parameters.

There are a few important points to appreciate about the fitting procedure used here. First, the data do not have to be rebinned to either linear or logarithmic wavelength scales; therefore all the information as well as the noise properties are preserved. Second, we do not need to perform continuum subtraction from either the object or the template, which is advantageous since that is always a poorly defined task; instead we rely on the continuum shape from the synthetic spectrum, with additional polynomial modifications (cf. Koleva et al. 2009 for a discussion of continuum fitting).

Figure 4 shows the result of the spectral fitting procedure applied to the co-added spectra of four of the stars in the Boötes I sample. Figure 5 shows the resulting velocity probability distribution for the same four stars. The velocity precision ranges from $0.25 \mathrm{~km} \mathrm{~s}^{-1}$ to $7 \mathrm{~km} \mathrm{~s}^{-1}$. For the brighter stars the probability distribution of the velocity is approximately Gaussian, while for the fainter star it is clearly asymmetric. For some of the faintest stars in our sample the probability distribution is even multi-modal.

\subsection{Application to Boötes I Stellar Spectra}

For each star, as a first step we apply our fitting procedure to the co-added spectra from each exposure. The purpose of using the co-added spectra here is primarily to determine the best-fit template for each object and the subsequent velocity estimate. Although this fitting is motivated by optimizing the radial velocity accuracy, and not primarily by measuring the 


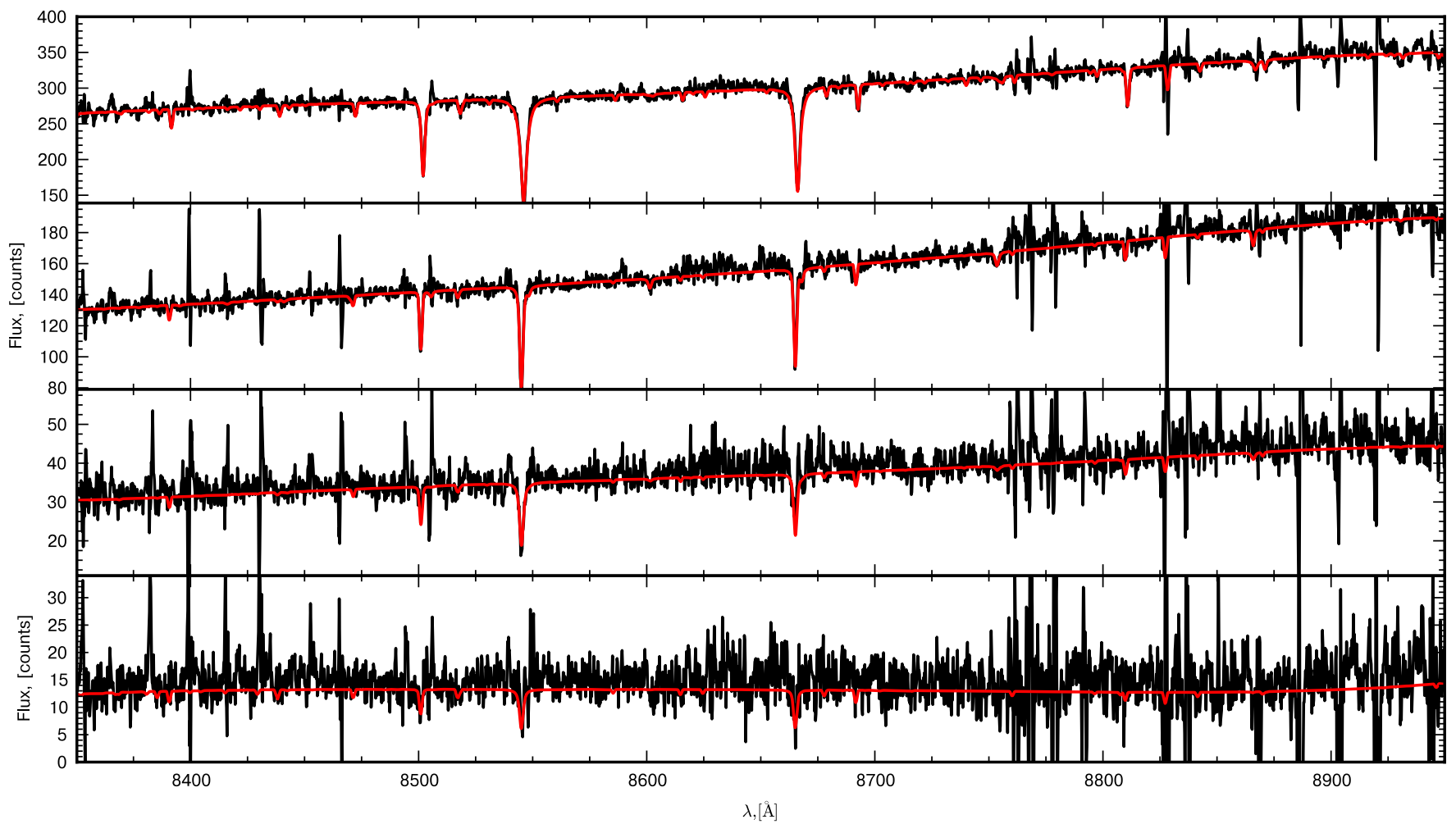

Figure 4. Examples of fitting synthetic model spectra to co-added spectra for several stars of different magnitudes (from top to bottom): SDSS J135922.59+143300.7 $(r=18.51)$, SDSS J140001.53+142154.2 $(r=19.35)$, SDSS J140002.44+142249.1 $(r=20.62)$, and SDSS J135951.07+143049.8 $(r=21.52)$. Black lines show the observed co-added spectra, while the red lines show the best-fit model spectra.

(A color version of this figure is available in the online journal.)

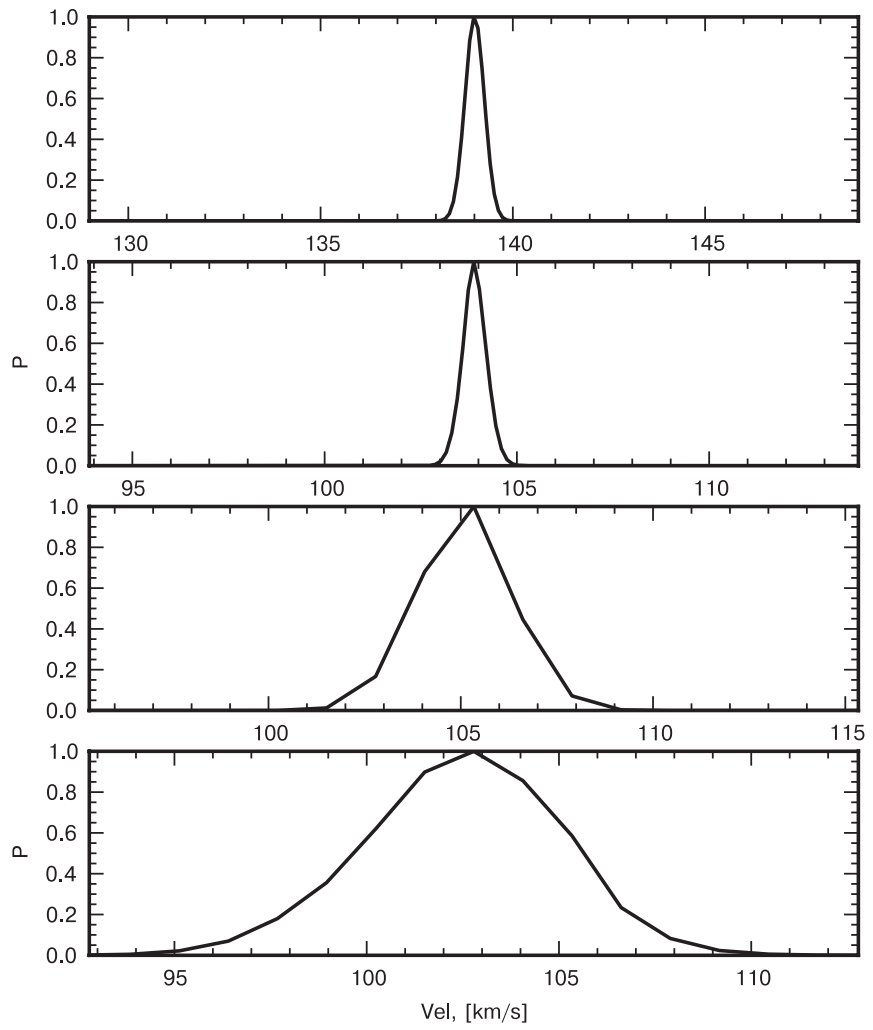

Figure 5. Un-normalized velocity posterior probability distributions derived from the co-added spectra, for the same four stars as shown in Figure 4. It is clear that, especially at faint magnitudes/lower signal to noise, the velocity probability distribution is not Gaussian. stellar astrophysical parameters $T_{\text {eff }}, \log (g)$, and $[\mathrm{Fe} / \mathrm{H}]$ from our template fit, the values of these parameters determined from the best-fit templates are reasonable, as illustrated by Figure 6 . This figure shows how the measured parameters correlate with radial velocities and colors of the stars. The left panel of Figure 6 shows the 2D distribution of radial velocities and metallicities of the stars. From the plot we see that the velocity peak at $100 \mathrm{~km} \mathrm{~s}^{-1}$ related to the Boötes I dwarf galaxy is produced by stars with $[\mathrm{Fe} / \mathrm{H}]<-1.5$, which is what we expect from the metallicity of Boötes I (Martin et al. 2007; Norris et al. 2008). In the middle panel of Figure 6 we show the 2D distribution of radial velocities and $\log (g)$. We see that the contamination (from foreground Milky Way stars) typically has high surface gravity $\log (g) \gtrsim 4.0$ - exactly what we expect from disk dwarfs. The right panel of the figure shows the correlation between determined effective temperature and $(g-r)$ color. Although of relatively low significance, a correlation still can clearly be seen. Overall we conclude that the parameters from the template fit are reasonably well determined using our fitting procedure and the adopted spectral library.

As the next step we use the best-fit templates as well as the initial velocity estimates for the final fitting of each individual (i.e., not co-added) observation of a given object in the same way as described above. The velocity estimate from the fit to the coadded spectra, plus or minus $50 \mathrm{~km} \mathrm{~s}^{-1}$, is used as a uniform prior on the radial velocity. That is, we make an assumption that the radial velocity of a given object in a single observation does not vary more than $50 \mathrm{~km} \mathrm{~s}^{-1}$ from the value measured from the co-added spectrum. From this step we obtain probability distributions of the velocities for each epoch observation of each object. We can use these repeated velocity measurements for several purposes: first, in order to assess our measurement 

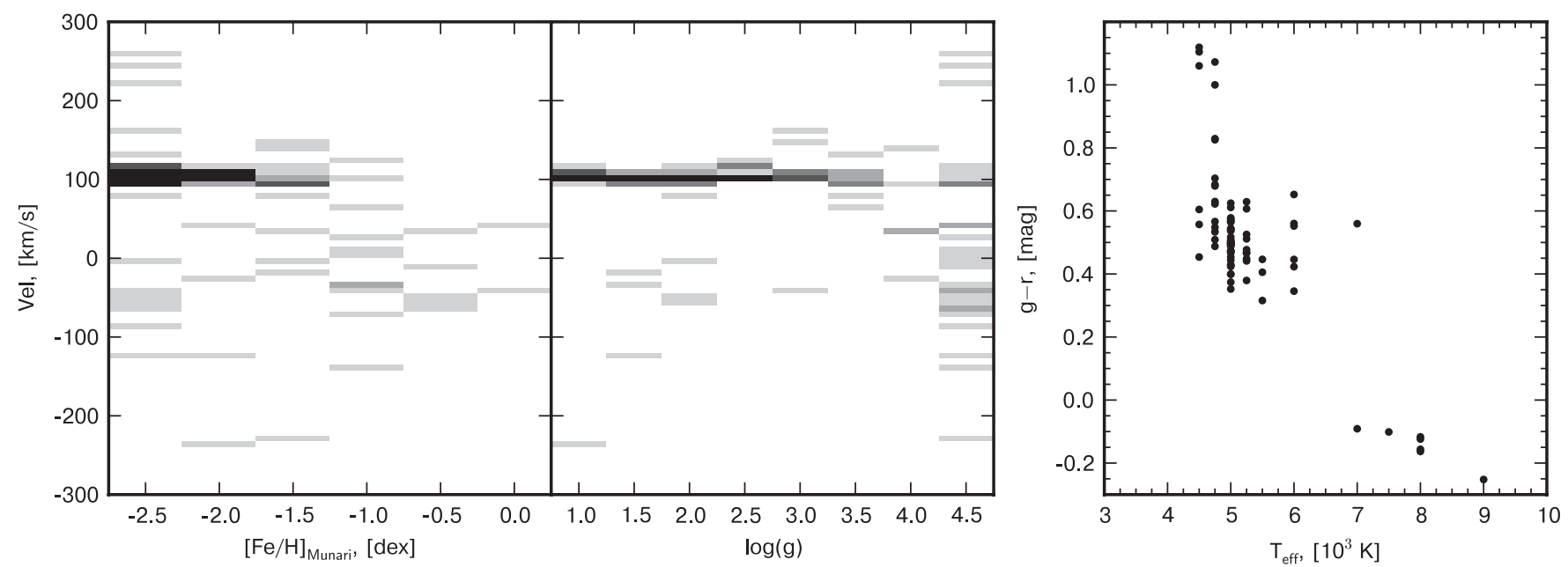

Figure 6. Stellar parameters determined from our synthetic spectrum template fit. The left panel shows the 2D histogram of radial velocities and metallicities of the best-fit templates for all observed stars. It is clear that the velocity peak at $\sim 100 \mathrm{~km} \mathrm{~s}^{-1}$, the systemic velocity of Boötes I, occurs at [Fe/H] $\lesssim-2$ dex, which is approximately the metallicity of Boötes I, while the background (halo, thin and thick disk) has $[\mathrm{Fe} / \mathrm{H}] \gtrsim-1.5$. The middle panel shows that most Boötes I stars have low surface gravities, as expected for giants, while the contaminating stars from the Milky Way foreground have almost exclusively $\log (g) \geqslant 4.0$, as expected for dwarfs. The right panel shows the correlation of the $g-r$ color vs. the effective temperature of the best-fit template. This correlation demonstrates that the effective temperatures are reasonably well determined.

errors; and second, to check for possible binarity/variability of the radial velocities (see Section 6). We end with a total sample of 112 stars for which we have derived radial velocities.

\subsection{Checking the Derived Uncertainties}

As described in the previous section, from our spectral fitting procedure applied to either individual or co-added spectra we determine the probability distribution of the radial velocity of a given star $\operatorname{Prob}(v)$, the MAP estimate of the velocity, and the error of that velocity. One of the most important checks on the validity of our results-as well as a check on the effectiveness of our reduction method-is the confirmation that the velocity errors that we determine from individual observations of specific (non-variable) stars are not systematically larger or smaller than the scatter between individual repeated measurements. That is, have we met our ambition of deriving correct and reliable uncertainties?

Figure 7 compares the standard deviation determined from repeated measurements to the mean error determined by our fitting procedure. In this plot we expect to see a one-to-one correlation, except for three cases: one, where there is intrinsic variability in the radial velocity; two, where there is some remaining systematic error which varies from observation to observation; or three, in the case where measurement errors from individual exposures are significantly larger than the onepixel resolution of the spectrograph $\left(\sim 15 \mathrm{~km} \mathrm{~s}^{-1}\right)$. In this latter case undersampling causes the probability distribution of the velocities to become significantly non-Gaussian, so the mean fitting error is an inadequate representation of possible variation of the velocities.

With reassuringly few exceptions, the data points in Figure 7 scatter about the one-to-one line. There is a slight apparent systematic tendency for the data points to be scattered more above the one-to-one line than below it, expected given that the error bars on the standard deviation are significantly asymmetric. The one-to-one relation extends in general down to a precision of a few hundred $\mathrm{m} \mathrm{s}^{-1}$, confirming that our derived error bars are reliable down to $0.1-0.2 \mathrm{~km} \mathrm{~s}^{-1}$. In the next section we show that several points lie above the one-to-one line not because we

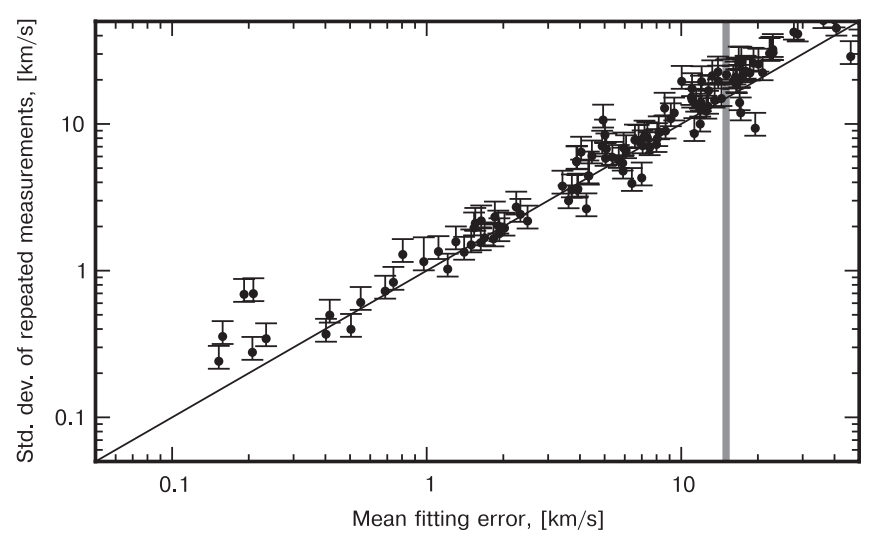

Figure 7. Comparison of the mean velocity error determined from the fitting procedure and the estimated standard deviation of the repeatedly measured velocities. Error bars show $68 \%$ confidence limits for the estimated standard deviations. The solid black line shows the one-to-one relation. The gray line shows the approximate velocity where the one-to-one relation between the mean error and the estimated standard deviation is expected to fail due to nonGaussianity of the probability distributions.

have underestimated our errors, but rather because of intrinsic variability of their radial velocities.

Since we were somewhat, albeit pleasantly, surprised at obtaining velocity precision substantially better than $1 \mathrm{~km} \mathrm{~s}^{-1}$, as confirmed by repeated measurements, we checked whether this may be some artifact of the algorithm due to the skylines. For example, if a systematically poorly subtracted skyline affecting a specific wavelength caused velocities to cluster near some fixed value, this situation could mimic small variances in repeated observations. A piece of circumstantial evidence against this hypothesis is that the nearly one-to-one relation of the error bars versus the standard deviation from repeated measurements (Figure 7) contains a set of stars with very different radial velocities, while it seems unlikely that skyline artifacts are present at all possible radial velocities. The most compelling evidence against this hypothesis is that, since we have radial velocity measurements spread over a month of observations, the variation of barycentric corrections is roughly $3 \mathrm{~km} \mathrm{~s}^{-1}$, 


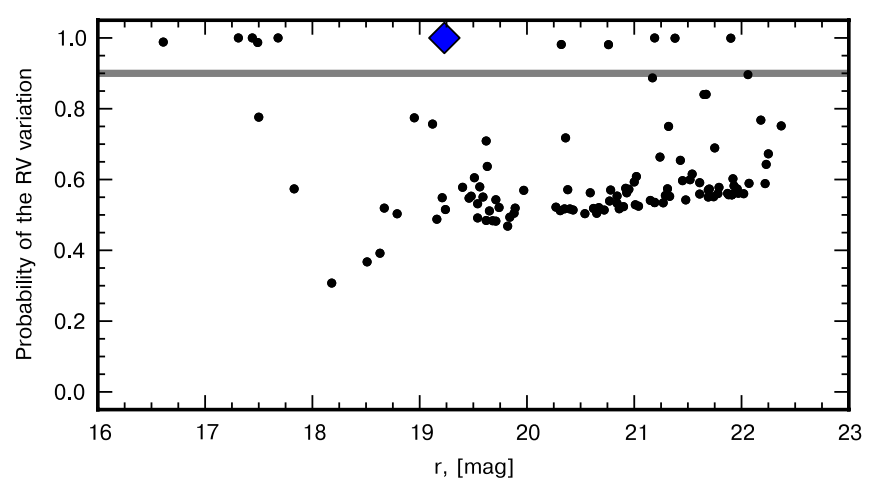

Figure 8. Probability of detected radial velocity variability for all 112 observed stars, computed using the Bayes factors for our two hypotheses: H1, there is no intrinsic variability in radial velocities; and $\mathrm{H} 2$, there is intrinsic variability with amplitude larger than $1 \mathrm{~km} \mathrm{~s}^{-1}$. The gray line shows the cut in Bayes factor, which is placed such that stars above the line favor the $\mathrm{H} 2$ hypothesis and are intrinsically variable. There are 11 such stars. The blue diamond indicates a known RR Lyrae star.

(A color version of this figure is available in the online journal.)

which is much larger than our claimed precision and scatters between repeated measurements; this would not be possible if our measurements were driven by some earth-velocity sky features or artifacts.

As a final cross-check of our radial velocity error bars, we compared them with the theoretical estimates of minimum possible velocity uncertainty, based on Fisher-matrix-like arguments (Murphy et al. 2008; Griest et al. 2010). This comparison suggests that our error bars are not unrealistic and are typically very close to that minimum velocity uncertainty, being above it only for a few of the faintest stars. This confirms that our velocity precision is not unreasonable.

A last remark is that, while the random errors seem to be correctly determined for the brightest stars with the highest precision $\left(<1 \mathrm{~km} \mathrm{~s}^{-1}\right.$ from individual exposures), there is a possibility that the systematic errors due to template mismatch (e.g., stellar spectra looking systematically different from spectra in our grid) are dominating the error budget.

\section{VARIABILITY ANALYSIS}

The goal of this section is to use our velocities, and velocity uncertainties, to estimate which of the observed stars show significant intrinsic variability, so that we may remove those stars from the analysis of the velocity distribution in Boötes I. We do this by testing the plausibility of two hypotheses for each object, the first hypothesis being that the object does not show significant variation in radial velocities, and the second hypothesis being that the object does show evidence for velocity variability with amplitude greater than $1 \mathrm{~km} \mathrm{~s}^{-1}$.

For each star and each of $N$ observations of each star our spectroscopic fitting procedure provides us with the probability distribution of the radial velocity $\operatorname{Prob}_{i}(v)$ associated with that observation. In general these probability distributions are not always Gaussian, especially in the low signal-to-noise cases, so in the following analysis we will avoid making any assumptions of Gaussianity.

For each star we assume that there is a certain intrinsic distribution of radial velocities $\Psi\left(v-v_{\text {sys }}\right)$, where $v_{\text {sys }}$ is the systemic velocity of the star. If the radial velocity of the source is not varying, then $\Psi\left(v-v_{\text {sys }}\right)$ is a delta function. The likelihood of the observations, assuming that they randomly sample $\Psi(v)$,
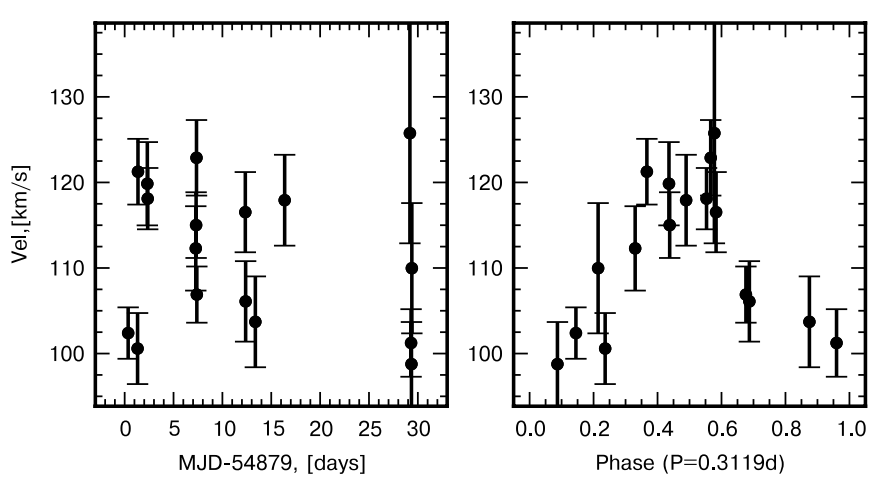

Figure 9. Radial velocity variation of the star SDSS J135951.33+143905.8, identified as an RR Lyrae variable by Dall'Ora et al. (2006) and Siegel (2006). The left panel shows the observed radial velocities as a function of the Julian date, while the right panel shows the observations phased with the photometric period $P=0.3119$ days from Siegel (2006).

can then be written as

$$
L=\prod_{i}^{N_{\mathrm{obs}}} \int \Psi\left(v-v_{\mathrm{sys}}\right) \operatorname{Prob}_{i}(v) d v .
$$

It is clear, for example, that if all the individual $\operatorname{Prob}_{i}(v)$ are Gaussians, and if the object's velocity is assumed to be constant, (i.e., $\Psi\left(v-v_{\text {sys }}\right)$ is a delta function), then the likelihood $L$ will be a Gaussian centered at the weighted mean of the centers of the Gaussians $\left(\operatorname{Prob}_{i}(v)\right)$, i.e., individual velocity measurements.

However, we do not make these Gaussian assumptions and can adopt a more general approach.

In order to assess the velocity variability of a given object we evaluate the likelihoods of our two hypotheses: H1, that the velocity of the object does not change, that is $\Psi\left(v-v_{\text {sys }}\right)=$ $\delta\left(v-v_{\text {sys }}\right)$; and $\mathrm{H} 2$, that the velocity is varying and the velocities are distributed uniformly between $v_{\text {sys }}-s$ and $v_{\text {sys }}+s$, where $s$ is the measure of the scatter:

$$
\Psi\left(v-v_{\mathrm{sys}}\right)=\left\{\begin{array}{ll}
\frac{1}{2 s} & \text { if }\left|v-v_{\mathrm{sys}}\right|<s \\
0 & \text { if }\left|v-v_{\mathrm{sys}}\right| \geqslant s
\end{array} .\right.
$$

For each hypothesis H1, H2 we can compute its likelihood using Equation (7), and marginalize over the parameters $v_{\text {sys }}$ and $s$, adopting uniform priors. The ratio of the likelihoods of two hypotheses marginalized over the parameters becomes a Bayes factor, $B$-which is a measure of the relative plausibility of the two hypotheses (Jeffreys 1961; Kass \& Raftery 1995; Trotta 2007). We calculate this for all 112 stars in our final sample.

Figure 8 shows the probabilities of variability calculated for all 112 of our stars, derived from Bayes factors, as a function of stellar apparent magnitude. The gray line shows the location of the probability threshold (see Kass \& Raftery 1995) which we use to identify 11 stars with significant evidence for radial velocity variation.

The blue diamond point, located significantly above the gray line, corresponds to one of our target stars. After (re-)discovering its variability, we realized that this star is a known RR Lyrae variable, from published photometric studies (Dall'Ora et al. 2006; Siegel 2006). This recovery does confirm that we can indeed correctly identify stars with variable radial velocity. Figure 9 shows the observed (as a function of observation time), and phased (using the photometric period) radial velocity variations for this RR Lyrae star.

Figure 10 illustrates the observed velocity data as a function of time for one bright, high $\mathrm{S} / \mathrm{N}$ star not detected to be velocity 

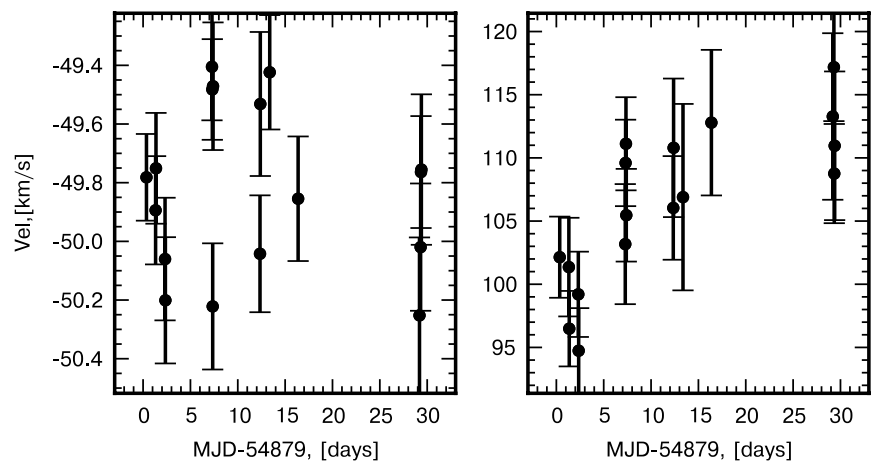

Figure 10. Radial velocity variations for two stars in our sample, one identified as variable, one not. Left panel: the radial velocity observations as a function of time for the fairly bright star in our sample, J135940.18+142428.0. Note the vertical scale. This is shown to illustrate the accuracy with which we are determining radial velocities. Right panel: radial velocity variation of star SDSS J140003.32+142851.4.

variable, and for another star with variability Bayes factors above our variability detection threshold.

In short, the discussion above, and the evidence in the shown in these figures, demonstrate that we are able to calculate a Bayes factor probability that each observed star is consistent with being radial velocity non-variable over the time in which we have observations. While passing this test says little about much longer period velocity variability, it does allow us to reliably identify stars for which we have radial velocity data, but whose kinematics cannot be included in a dynamical analysis at face value. Applying that criterion, we restrict further analysis to 100 stars, with adequate velocity measurements and error bars, and no evidence for velocity variability.

\section{COMPARISON WITH PUBLISHED KINEMATIC STUDIES OF BOÖTES I}

Two observational kinematic studies of Boötes I (Muñoz et al. 2006; Martin et al. 2007), and two other analyses (Wolf et al. 2010; Norris et al. 2010a, 2010b), have been published, providing information which we may compare with our results.

Muñoz et al. (2006) used the WIYN telescope and the Hydra multifiber spectrograph to measure radial velocities for 58 candidate member stars, all brighter than $g=19$. Considering the strength of the Mgb absorption features, they classify fully 30 of these as giants. Further considering position, velocity, and line strength, they identify seven stars located within 10 arcmin of the center of Boötes I to define the mean velocity and velocity dispersion of Boötes I, finding a mean velocity $95.6 \pm$ $3.4 \mathrm{~km} \mathrm{~s}^{-1}$ and central velocity dispersion $6.6 \pm 2.3 \mathrm{~km} \mathrm{~s}^{-1}$. With these values they further identify a total of 12 stars as "potential $3 \sigma$ members," 11 being within a radius of 15 arcmin of the center of Boötes I, the 12th at 27 arcmin distance (the half-light radius is 12.5 arcmin). These 12 candidate members provide a higher mean velocity, $98.4 \pm 2.9 \mathrm{~km} \mathrm{~s}^{-1}$, and a higher dispersion of $\sigma_{0}=9.0 \pm 2.2 \mathrm{~km} \mathrm{~s}^{-1}$; this higher dispersion, as the authors note "corroborat[es] the apparent increase of the velocity dispersion with radius" (Muñoz et al. 2006, p. L53). The authors also note that they have two further "likely highvelocity members." The full set of 14 stars provides a velocity dispersion of $\sigma_{0}=14.6 \pm 3.0 \mathrm{~km} \mathrm{~s}^{-1}$, suggesting "a possibly dramatic increase of the Boötes Ivelocity dispersion with radius" (p. L53). The 12-member-star sample, with its Boötes I velocity dispersion of $\sigma_{0}=9.0 \pm 2.2 \mathrm{~km} \mathrm{~s}^{-1}$, is that adopted by Wolf et al. (2010) in their dynamical analysis. We compare our results for nine stars in common in the left panel of Figure 11. Our results in this study are inconsistent with the velocity dispersions reported by Muñoz et al. (2006), and we rule out their suggested rapid radial increase in velocity dispersion (see below).

Martin et al. (2007) observed 96 candidate Boötes I red giants from SDSS (DR4) with Keck/DEIMOS. They identified a sample of 24 member stars, each with a radial velocity determined with an accuracy smaller than $6 \mathrm{~km} \mathrm{~s}^{-1}$ (cf. Martin et al. 2007 for details). From this sample they find a mean velocity of $99.9 \pm 2.4 \mathrm{~km} \mathrm{~s}^{-1}$, with central velocity dispersion $\sigma=6.5_{-1.3}^{+2.1} \mathrm{~km} \mathrm{~s}^{-1}$ for their final sample of 24 stars with small velocity uncertainties. We observed, by design, 27 stars in common with the Martin et al. sample.

We compare our measurements for the 27 stars in common with the radial velocity measurements by Martin et al. (2007) in the rightmost two panels of Figure 11. The zero points of our radial velocity measurements are consistent. The mean velocities, for the common 27-member-star sample, are $\bar{V}_{\text {keck }}=$ $99.6 \pm 1.7 \mathrm{~km} \mathrm{~s}^{-1}$, and $\bar{V}_{\mathrm{VLT}, 27}=101.2 \pm 2.0 \mathrm{~km} \mathrm{~s}^{-1}$, in excellent agreement. The mean velocity from our full sample is $\bar{V}_{\mathrm{VLT}}=101.8 \pm 0.7 \mathrm{~km} \mathrm{~s}^{-1}$. The right-hand panel of Figure 11 compares our derived velocity errors with those reported by Martin et al. (2007). It is apparent that our derived singlevelocity errors are a factor of about two smaller than those of Martin et al. (2007), at high S/N in both studies. We may also do a very crude check on the accuracy of the quoted velocity uncertainties. Comparing stars in common with Martin et al. (2007), the velocity difference, in units normalized by the quadrature sum of our quoted errors and those of Martin et al. (2007), has a sigma of 2.7. This is robust evidence that the combined errors are underestimated. The results of Figure 7 suggest that we have correctly calculated our uncertainties. In addition, as we discuss in the section below, our derived velocity dispersion for Boötes I is significantly below that published by other studies. We interpret this difference, in essence a difference between fiber-fed and slit spectrographs, as reflecting the inherent precision limits of velocity determination using multi-slit spectrographs.

For completeness, we note the studies of Boötes I by Norris et al. (2010a, 2010b). Norris et al. (2010a) obtained spectra of candidate RGB members within a 1 deg radius of Boötes I, using the AAT+AAOmega facility. Their study was designed to identify candidate members of Boötes I at large distances from the center, for analysis of the chemical abundance distribution, and to allow subsequent more detailed study for kinematics, so they had, by design, relatively low-velocity precision $\left(10 \mathrm{~km} \mathrm{~s}^{-1}\right)$. Nonetheless, they identify candidate members out to $60 \mathrm{arcmin}$ (five halflight radii, cf. their Figure 6) from the center of Boötes I. Norris et al. (2010a) observed five stars in common with Martin et al. (2007) and report agreement in mean velocity within $3 \mathrm{~km} \mathrm{~s}^{-1}$ and a dispersion in velocity differences for those five stars of $2.3 \mathrm{~km} \mathrm{~s}^{-1}$ (in spite of their suggested $10 \mathrm{~km} \mathrm{~s}^{-1}$ accuracy). They further report work in preparation confirming one of their candidates (Boo-980) to be an extremely metalpoor giant at 3.9 half-light radii from the center of Boötes I, and with radial velocity $99.0 \pm 0.5 \mathrm{~km} \mathrm{~s}^{-1}$. Norris et al. (2010b) report on a follow-up high dispersion UVES study of one star identified by Norris et al. (2010a). This star, Boo-1137, is also extremely metal-poor. Of relevance here, it lies 24 arcmin (two half-light radii) from the center of Boötes I and has a radial velocity $V_{\text {uves }}=99.1 \pm 0.5 \mathrm{~km} \mathrm{~s}^{-1}$. Both of these velocities are in remarkable agreement with the mean velocity of the Boötes I 

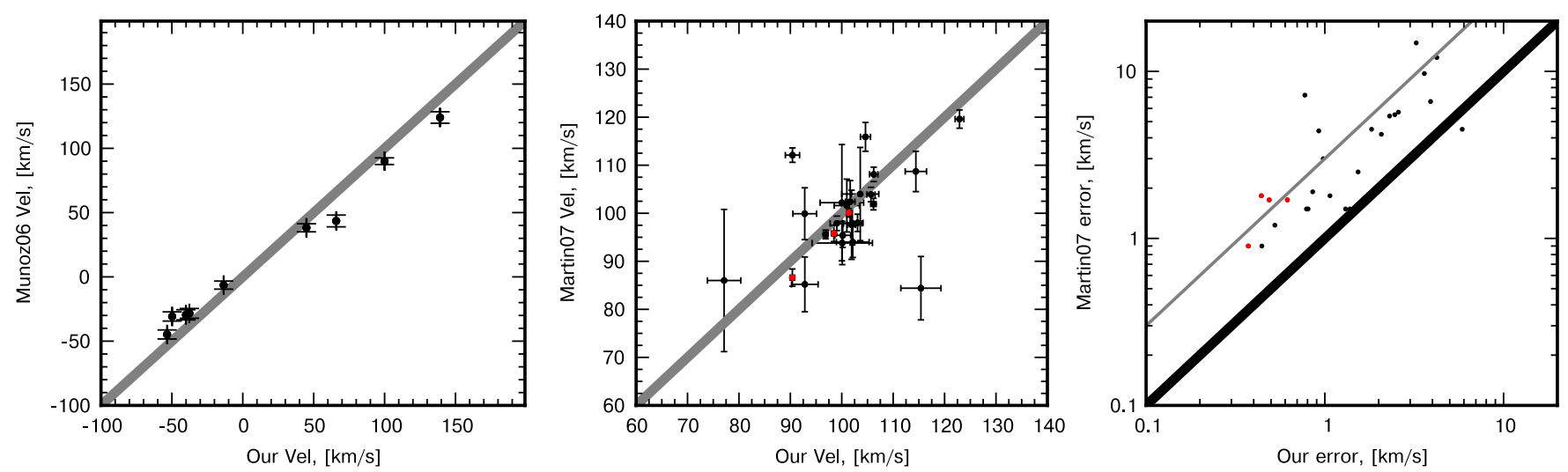

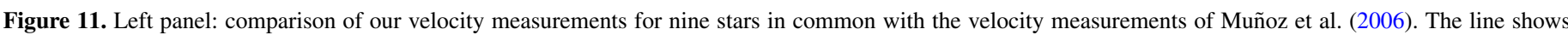

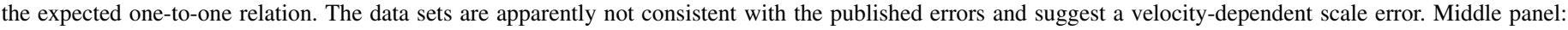

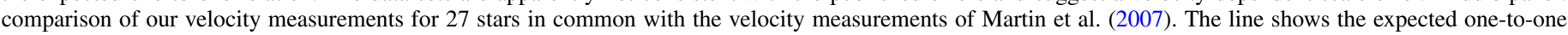

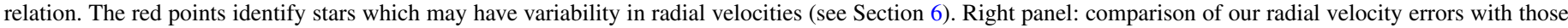
of Martin et al. (2007). The thick black line shows the one-to-one relation, while the gray line shows the $y=3 x$ relation.

(A color version of this figure is available in the online journal.)

system, considering their large Boötes-centric distance, and hint at a low-velocity dispersion in the outer very metal-poor populations in Boötes I.

\section{THE VELOCITY DISPERSION(S) IN BOÖTES I}

Equation (7) gives us a way to determine the best velocity estimate from several velocity measurements. After removing those stars that we suspect to be variable in radial velocity, we model the remaining 100 star sample of velocities assuming that each velocity is constant (i.e., $\Psi\left(v-v_{\text {sys }}\right)$ is a delta-function). Thus, we derive the probability distribution of the systemic velocity for each star by multiplying radial velocity probability distributions from separate measurements $\operatorname{Prob}(v)=\prod_{\text {observations }} \operatorname{Prob}_{i}(v)$. Those probability distributions are in most cases very close to Gaussians, so in the following analysis we assume that we have the velocity estimate and the Gaussian error bar for each star. Table 1 lists those velocities, and other information, for each star.

Figure 12 shows our resulting velocity distributions for two different subsets of stars. The top panel shows the distribution of velocities for the 37 stars which are likely members, i.e., those with $\log (g)<3.5,[\mathrm{Fe} / \mathrm{H}]<-1.5$, not showing any variability according to our Bayes factor criterion and with small errors of their radial velocity measurement $\sigma_{v}<2.5 \mathrm{~km} \mathrm{~s}^{-1}$. The bottom panel shows the corresponding velocity distribution for all 100 non-velocity variable stars in our sample. The velocity distributions illustrate two important points. First, the velocity peak due to Boötes I member stars is quite evident. Second, it is clear that the velocity dispersion of the stars in Boötes I is significantly smaller than $6 \mathrm{~km} \mathrm{~s}^{-1}$, or $9 \mathrm{~km} \mathrm{~s}^{-1}$, the global dispersions measured previously (Muñoz et al. 2006; Martin et al. 2007). In the top panel especially, the bulk of the distribution looks similar to a Gaussian with $\sim 3 \mathrm{~km} \mathrm{~s}^{-1}$ dispersion, while in the bottom panel the less restrictively selected sample shows, in addition to the low dispersion "core," rather pronounced higher-velocity tails.

In order to assess the distribution of radial velocities we fit the observed velocity distribution with two different models: one where the velocity distribution in the galaxy is represented by a single Gaussian, and the second with the velocity distribution being the sum of two Gaussians with the same mean. To perform these fits, we follow the standard Bayesian approach. We write down the probability distribution as a function of the template metallicity, template $\log (g)$, and radial velocity as the mixture of distributions for the background (with fraction $f_{\mathrm{bg}}$ and for Boötes I):

$$
\begin{gathered}
P\left(\left[\frac{\mathrm{Fe}}{\mathrm{H}}\right], \log (g), v\right)=f_{\mathrm{bg}} P_{\mathrm{bg}}\left[\frac{\mathrm{Fe}}{\mathrm{H}}\right] P_{\mathrm{bg}}(\log (g)) P_{\mathrm{bg}}(v) \\
+\left(1-f_{\mathrm{bg}}\right) P_{\mathrm{boo}}\left[\frac{\mathrm{Fe}}{\mathrm{H}}\right] P_{\mathrm{boo}}(\log (g)) P_{\mathrm{boo}}(v) .
\end{gathered}
$$

This technique minimizes the subjectivity involved in our selection, for illustrative purposes, of the 37-member-star sub-sample noted above, where we subjectively imposed an astrophysical prior, and which we restricted to the small subset of highest precision data.

We assume that the probability distributions $P_{\mathrm{bg}}\left[\frac{\mathrm{Fe}}{\mathrm{H}}\right]$, $P_{\mathrm{bg}}(\log (g)), P_{\mathrm{bg}}(v), P_{\mathrm{boo}}\left[\frac{\mathrm{Fe}}{\mathrm{H}}\right]$, and $P_{\mathrm{bg}}(\log (g))$ are Gaussians with different means and standard deviations. For the radial velocity distribution of stars in the dwarf $P_{\text {boo }}(v)$, we assume that it is either a single Gaussian or the sum of two Gaussians with different dispersions but the same mean. Having the probability distribution defined in Equation (9) we perform a standard Markov Chain Monte Carlo (MCMC) sampling of the available parameter space in order to determine the posterior probability distribution for the parameters of $P_{\mathrm{boo}}(v)$. We use the standard Metropolis-Hastings algorithm (Metropolis et al. 1953; Hastings 1970) implemented in the pymc package (Patil et al. 2010); see, e.g., Neal (1993) for a review of the MCMC method.

The posterior probability distribution for the velocity dispersion of Boötes I for the single-Gaussian hypothesis is shown in Figure 13. The velocity dispersion estimate is then $4.6_{-0.6}^{+0.8} \mathrm{~km} \mathrm{~s}^{-1}$. This Gaussian dispersion is shown overlaid on the kinematic data in the middle panel. To graphically illustrate that our results are robust against sample selection, in the bottom panel we show the same Gaussian distribution overlaid on the subset of 37 high-probability members with excellent data. The fit is acceptable, but far from excellent.

If we make the assumption that the Boötes I velocity distribution consists of two Gaussians (in addition to the fit to the background), where one Gaussian has higher dispersion than the other, the posterior probability distributions for the velocity dispersions of the two components are those shown 
Table 1

Observational Data for All Stars Observed

\begin{tabular}{|c|c|c|c|c|c|c|c|c|c|c|c|c|}
\hline ID & Name & $\begin{array}{c}\alpha \\
(\mathrm{deg})\end{array}$ & $\begin{array}{c}\delta \\
(\mathrm{deg})\end{array}$ & $\begin{array}{c}g \\
(\mathrm{mag})\end{array}$ & $\begin{array}{c}r \\
(\mathrm{mag})\end{array}$ & $\begin{array}{c}\mathrm{RV} \\
\left(\mathrm{km} \mathrm{s}^{-1}\right)\end{array}$ & $\begin{array}{l}\text { RV Error } \\
\left(\mathrm{km} \mathrm{s}^{-1}\right)\end{array}$ & $P_{\mathrm{var}}$ & $\begin{array}{l}T_{\text {eff }} \\
(\mathrm{K})\end{array}$ & {$[\mathrm{Fe} / \mathrm{H}]$} & $\log (g)$ & Best flag \\
\hline 0 & $\mathrm{~J} 135921.36+143606.3$ & 209.8390 & 14.6017 & 17.8 & 17.0 & -13.50 & 0.10 & 0.57 & 4750 & -0.5 & 4.5 & \\
\hline 1 & J135922.59+143300.7 & 209.8442 & 14.5502 & 19.0 & 18.5 & 139.12 & 0.35 & 0.77 & 5250 & -1.5 & 4.0 & \\
\hline 2 & J135933.50+142821.6 & 209.8896 & 14.4727 & 21.9 & 21.5 & 115.40 & 3.91 & 0.56 & 5000 & -2.5 & 2.5 & \\
\hline 3 & J135934.36+143017.1 & 209.8932 & 14.5048 & 22.0 & 21.5 & 77.11 & 3.25 & 0.56 & 4000 & -2.5 & 3.5 & \\
\hline 4 & J135934.77+143503.4 & 209.8949 & 14.5843 & 21.2 & 20.7 & -228.29 & 1.36 & 0.54 & 5000 & -1.5 & 4.5 & \\
\hline 5 & J135935.66+143735.0 & 209.8986 & 14.6264 & 20.0 & 19.4 & -19.40 & 0.44 & 0.57 & 4750 & -1.5 & 1.5 & \\
\hline 6 & J135937.60+142647.6 & 209.9067 & 14.4466 & 21.6 & 21.1 & 103.23 & 2.54 & 0.59 & 5000 & -2.5 & 1.0 & \\
\hline 7 & J135939.36+142638.4 & 209.9140 & 14.4440 & 20.4 & 19.9 & 101.91 & 0.77 & 0.57 & 5250 & -2.0 & 2.5 & B \\
\hline 8 & J135940.18+142428.0 & 209.9174 & 14.4078 & 17.5 & 16.4 & -49.80 & 0.05 & 0.78 & 4500 & -0.5 & 4.5 & \\
\hline 9 & $\mathrm{~J} 135940.66+142712.0$ & 209.9195 & 14.4533 & 22.1 & 21.7 & $\mathrm{X}$ & $\mathrm{X}$ & $\mathrm{X}$ & $\mathrm{X}$ & $\mathrm{X}$ & $\mathrm{X}$ & \\
\hline 10 & $\mathrm{~J} 135941.78+144035.2$ & 209.9241 & 14.6765 & 22.2 & 21.9 & $\mathrm{X}$ & $\mathrm{X}$ & $\mathrm{X}$ & $\mathrm{X}$ & $\mathrm{X}$ & $\mathrm{X}$ & \\
\hline 11 & $\mathrm{~J} 135942.18+142942.2$ & 209.9258 & 14.4951 & 19.6 & 19.0 & 90.34 & 0.38 & 0.48 & 5000 & -2.5 & 1.5 & $\mathrm{~B}$ \\
\hline 12 & $\mathrm{~J} 135943.12+144054.1$ & 209.9297 & 14.6817 & 21.2 & 20.6 & -137.63 & 2.38 & 0.89 & 6000 & -1.0 & 4.5 & \\
\hline 13 & $\mathrm{~J} 135943.43+143438.3$ & 209.9310 & 14.5773 & 20.7 & 20.3 & 99.81 & 1.16 & 0.51 & 5000 & -2.5 & 1.0 & B \\
\hline 14 & J135944.57+143709.6 & 209.9358 & 14.6193 & 20.4 & 19.9 & 97.93 & 0.87 & 0.52 & 4750 & -2.5 & 1.0 & $\mathrm{~B}$ \\
\hline 15 & $\mathrm{~J} 135944.70+142601.8$ & 209.9363 & 14.4338 & 21.6 & 20.9 & $\mathrm{X}$ & $\mathrm{X}$ & $\mathrm{X}$ & $\mathrm{X}$ & $\mathrm{X}$ & $\mathrm{X}$ & \\
\hline 16 & $\mathrm{~J} 135944.95+143230.1$ & 209.9373 & 14.5417 & 20.8 & 20.3 & 103.07 & 1.07 & 0.54 & 5250 & -2.0 & 2.5 & $\mathrm{~B}$ \\
\hline 17 & $\mathrm{~J} 135945.06+142327.3$ & 209.9378 & 14.3909 & 21.2 & 20.7 & 102.84 & 1.55 & 0.66 & 5000 & -2.0 & 2.5 & B \\
\hline 18 & $\mathrm{~J} 135945.71+142552.5$ & 209.9405 & 14.4313 & 22.2 & 21.9 & 100.09 & 5.87 & 0.67 & 5000 & -2.5 & 2.0 & \\
\hline 19 & $\mathrm{~J} 135945.72+142230.9$ & 209.9405 & 14.3753 & 21.7 & 21.2 & 91.91 & 2.76 & 0.57 & 5000 & -2.5 & 4.5 & \\
\hline 20 & $\mathrm{~J} 135946.26+143409.2$ & 209.9428 & 14.5692 & 19.9 & 19.4 & 143.52 & 0.55 & 0.49 & 5250 & -1.5 & 3.0 & \\
\hline 21 & $\mathrm{~J} 135946.33+142511.8$ & 209.9431 & 14.4200 & 20.4 & 19.9 & 98.12 & 0.83 & 0.72 & 5000 & -2.5 & 2.0 & $\mathrm{~B}$ \\
\hline 22 & $\mathrm{~J} 135947.06+142852.5$ & 209.9461 & 14.4813 & 21.5 & 21.1 & 100.99 & 2.46 & 0.54 & 5000 & -2.0 & 1.5 & B \\
\hline 23 & $\mathrm{~J} 135947.57+142334.5$ & 209.9482 & 14.3929 & 17.7 & 16.6 & 36.75 & 0.15 & 1.00 & 4500 & -0.5 & 4.0 & \\
\hline 24 & J135948.14+143646.6 & 209.9506 & 14.6130 & 20.9 & 20.3 & 103.20 & 1.27 & 0.52 & 5000 & -2.0 & 1.5 & $\mathrm{~B}$ \\
\hline 25 & $\mathrm{~J} 135948.33+143203.5$ & 209.9514 & 14.5343 & 19.7 & 19.3 & 106.17 & 0.53 & 0.51 & 5500 & -2.0 & 1.0 & $\mathrm{~B}$ \\
\hline 26 & J135948.53+144204.4 & 209.9522 & 14.7012 & 20.3 & 19.8 & 111.75 & 2.08 & 0.98 & 5000 & -2.0 & 2.0 & \\
\hline 27 & J135948.96+142428.4 & 209.9540 & 14.4079 & 20.9 & 20.4 & -34.96 & 1.07 & 0.52 & 5250 & -1.0 & 4.5 & \\
\hline 28 & J135950.13+141944.4 & 209.9589 & 14.3290 & 20.3 & 19.7 & 101.74 & 0.94 & 0.52 & 5000 & -2.0 & 2.5 & $\mathrm{~B}$ \\
\hline 29 & $\mathrm{~J} 135950.75+143114.2$ & 209.9615 & 14.5206 & 21.8 & 21.3 & 92.81 & 2.58 & 0.69 & 5000 & -2.0 & 1.5 & \\
\hline 30 & $\mathrm{~J} 135950.91+143002.7$ & 209.9621 & 14.5008 & 17.5 & 16.5 & 99.95 & 0.09 & 0.99 & 4750 & -1.5 & 1.5 & \\
\hline 31 & J135951.07+143049.8 & 209.9628 & 14.5138 & 22.0 & 21.5 & 102.14 & 3.18 & 0.57 & 4000 & -2.5 & 1.0 & \\
\hline 32 & $\mathrm{~J} 135951.33+143905.8$ & 209.9639 & 14.6516 & 19.3 & 19.3 & 111.07 & 1.98 & 1.00 & 7000 & -2.0 & 2.5 & \\
\hline 33 & $\mathrm{~J} 135951.70+143543.3$ & 209.9655 & 14.5954 & 19.4 & 18.8 & 99.94 & 0.30 & 0.58 & 4500 & -2.5 & 1.0 & B \\
\hline 34 & J135951.87+143018.9 & 209.9662 & 14.5053 & 21.3 & 20.8 & 35.47 & 1.67 & 0.53 & 5000 & -1.5 & 4.0 & \\
\hline 35 & $\mathrm{~J} 135952.11+144039.3$ & 209.9672 & 14.6776 & 21.4 & 21.0 & 82.79 & 8.22 & 1.00 & 6000 & -2.5 & 4.5 & \\
\hline 36 & J135952.33+143245.6 & 209.9681 & 14.5460 & 20.7 & 20.2 & 99.05 & 1.31 & 0.52 & 5000 & -2.0 & 1.0 & $\mathrm{~B}$ \\
\hline 37 & $\mathrm{~J} 135953.00+142232.1$ & 209.9709 & 14.3756 & 22.2 & 21.8 & 102.67 & 5.89 & 0.64 & 5000 & -2.5 & 3.5 & \\
\hline 38 & $\mathrm{~J} 135953.12+142734.3$ & 209.9713 & 14.4595 & 20.8 & 20.3 & 104.70 & 1.28 & 0.53 & 5000 & -2.5 & 1.5 & B \\
\hline 39 & J135953.75+143055.9 & 209.9740 & 14.5156 & 20.7 & 20.2 & 122.91 & 0.86 & 0.50 & 5000 & -1.0 & 2.5 & \\
\hline 40 & J135953.93+142951.6 & 209.9747 & 14.4977 & 22.1 & 21.7 & -235.57 & 4.39 & 0.59 & 4000 & -2.0 & 1.0 & \\
\hline 41 & $\mathrm{~J} 135954.41+144244.2$ & 209.9767 & 14.7123 & 21.2 & 20.7 & -45.51 & 6.79 & 1.00 & 5000 & -2.5 & 4.5 & \\
\hline 42 & $\mathrm{~J} 135954.89+143715.3$ & 209.9787 & 14.6209 & 19.5 & 19.6 & 109.80 & 1.68 & 0.55 & 8000 & -2.0 & 3.5 & \\
\hline 43 & $\mathrm{~J} 135955.33+143452.8$ & 209.9806 & 14.5813 & 19.7 & 19.1 & 100.03 & 0.38 & 0.48 & 5000 & -2.0 & 1.5 & B \\
\hline 44 & $\mathrm{~J} 135955.98+143425.6$ & 209.9833 & 14.5738 & 19.6 & 19.1 & 114.36 & 0.38 & 0.58 & 5000 & -1.5 & 2.5 & \\
\hline 45 & J135956.41+143556.9 & 209.9851 & 14.5992 & 17.3 & 16.3 & -37.63 & 0.08 & 1.00 & 4750 & 0.0 & 4.5 & \\
\hline 46 & J135956.43+142057.4 & 209.9852 & 14.3493 & 19.2 & 18.7 & -72.15 & 0.33 & 0.55 & 5250 & -1.0 & 4.5 & \\
\hline 47 & J135956.71+142516.3 & 209.9863 & 14.4212 & 19.8 & 19.2 & 13.06 & 0.27 & 0.47 & 4750 & -1.0 & 4.5 & \\
\hline 48 & J135957.84+142802.5 & 209.9910 & 14.4674 & 20.4 & 19.9 & 101.64 & 0.92 & 0.52 & 5250 & -2.0 & 3.5 & \\
\hline 49 & $\mathrm{~J} 135958.70+144040.2$ & 209.9946 & 14.6779 & 22.3 & 21.8 & 107.88 & 6.46 & 0.59 & 5000 & -2.5 & 1.0 & \\
\hline 50 & J135959.70+143633.1 & 209.9988 & 14.6092 & 20.9 & 20.4 & 224.82 & 7.09 & 0.58 & 7000 & -2.5 & 4.5 & \\
\hline 51 & $\mathrm{~J} 140000.24+143234.9$ & 210.0010 & 14.5430 & 20.6 & 20.1 & 108.27 & 0.89 & 0.56 & 5000 & -2.0 & 1.0 & $\mathrm{~B}$ \\
\hline 52 & $\mathrm{~J} 140000.75+143529.0$ & 210.0031 & 14.5914 & 19.7 & 20.0 & 99.52 & 2.69 & 0.54 & 9000 & -1.5 & 3.5 & \\
\hline 53 & $\mathrm{~J} 140000.99+143126.7$ & 210.0041 & 14.5241 & 21.8 & 21.5 & 110.49 & 3.12 & 0.56 & 6000 & -1.5 & 3.0 & \\
\hline 54 & $\mathrm{~J} 140001.42+143424.1$ & 210.0059 & 14.5734 & 20.6 & 20.1 & -28.59 & 0.92 & 0.50 & 5500 & -2.0 & 4.0 & \\
\hline 55 & $\mathrm{~J} 140001.53+142154.2$ & 210.0064 & 14.3651 & 19.9 & 19.4 & 103.50 & 0.44 & 0.52 & 4750 & -2.5 & 1.0 & B \\
\hline 56 & $\mathrm{~J} 140001.66+142454.8$ & 210.0069 & 14.4152 & 19.2 & 18.6 & 93.97 & 0.26 & 0.52 & 5000 & -1.5 & 4.5 & \\
\hline 57 & $\mathrm{~J} 140002.23+144114.2$ & 210.0093 & 14.6873 & 20.9 & 20.3 & 79.61 & 1.25 & 0.57 & 4750 & -1.5 & 2.0 & \\
\hline 58 & $\mathrm{~J} 140002.28+142653.4$ & 210.0095 & 14.4482 & 22.0 & 21.6 & 103.60 & 3.60 & 0.56 & 5000 & -2.5 & 2.5 & \\
\hline 59 & $\mathrm{~J} 140002.44+142249.1$ & 210.0102 & 14.3803 & 21.2 & 20.6 & 101.64 & 1.55 & 0.54 & 5000 & -2.0 & 3.0 & B \\
\hline 60 & $\mathrm{~J} 140003.07+143023.6$ & 210.0128 & 14.5066 & 21.5 & 21.0 & 102.06 & 1.83 & 0.60 & 5000 & -2.5 & 1.0 & $\mathrm{~B}$ \\
\hline 61 & J140003.32+142851.4 & 210.0138 & 14.4810 & 20.7 & 20.2 & 105.36 & 1.67 & 0.98 & 4750 & -2.5 & 1.5 & \\
\hline 62 & $\mathrm{~J} 140003.47+143952.2$ & 210.0145 & 14.6645 & 21.7 & 21.3 & 103.90 & 4.00 & 0.55 & 6000 & -2.0 & 3.0 & \\
\hline 63 & $\mathrm{~J} 140005.16+143427.8$ & 210.0215 & 14.5744 & 20.9 & 20.5 & 104.05 & 1.39 & 0.56 & 5000 & -2.5 & 2.0 & $\mathrm{~B}$ \\
\hline
\end{tabular}


Table 1

(Continued)

\begin{tabular}{|c|c|c|c|c|c|c|c|c|c|c|c|c|}
\hline ID & Name & $\begin{array}{c}\alpha \\
(\mathrm{deg})\end{array}$ & $\begin{array}{c}\delta \\
(\mathrm{deg})\end{array}$ & $\begin{array}{c}g \\
(\mathrm{mag})\end{array}$ & $\begin{array}{c}r \\
(\mathrm{mag})\end{array}$ & $\begin{array}{c}\mathrm{RV} \\
\left(\mathrm{km} \mathrm{s}^{-1}\right)\end{array}$ & $\begin{array}{l}\text { RV Error } \\
\left(\mathrm{km} \mathrm{s}^{-1}\right)\end{array}$ & $P_{\mathrm{var}}$ & $\begin{array}{l}T_{\text {eff }} \\
(\mathrm{K})\end{array}$ & {$[\mathrm{Fe} / \mathrm{H}]$} & $\log (g)$ & Best flag \\
\hline 64 & $\mathrm{~J} 140005.33+143023.3$ & 210.0222 & 14.5065 & 21.0 & 20.6 & 100.19 & 1.53 & 0.52 & 5000 & -2.5 & 1.0 & B \\
\hline 65 & $\mathrm{~J} 140005.61+142618.8$ & 210.0234 & 14.4386 & 20.4 & 19.9 & 106.22 & 0.81 & 0.51 & 5000 & -2.5 & 1.5 & B \\
\hline 66 & $\mathrm{~J} 140008.67+143654.3$ & 210.0361 & 14.6151 & 20.9 & 20.4 & 103.07 & 1.23 & 0.55 & 5000 & -2.5 & 1.0 & B \\
\hline 67 & $\mathrm{~J} 140010.30+142626.5$ & 210.0429 & 14.4407 & 21.9 & 21.6 & 102.33 & 3.48 & 0.58 & 5000 & -2.5 & 1.0 & \\
\hline 68 & $\mathrm{~J} 140010.61+143823.8$ & 210.0442 & 14.6400 & 19.9 & 19.4 & 101.19 & 0.52 & 0.50 & 5000 & -2.0 & 2.0 & B \\
\hline 69 & $\mathrm{~J} 140010.69+142924.4$ & 210.0446 & 14.4901 & 19.7 & 19.2 & 103.55 & 0.34 & 0.48 & 4500 & -2.5 & 1.0 & B \\
\hline 70 & $\mathrm{~J} 140011.53+142556.0$ & 210.0480 & 14.4322 & 21.5 & 21.0 & 114.41 & 2.07 & 0.62 & 5000 & -2.5 & 1.0 & $\mathrm{~B}$ \\
\hline 71 & $\mathrm{~J} 140012.23+142922.0$ & 210.0510 & 14.4894 & 21.3 & 20.9 & 91.42 & 2.61 & 0.75 & 6000 & -2.0 & 4.5 & \\
\hline 72 & $\mathrm{~J} 140012.41+143327.3$ & 210.0517 & 14.5576 & 20.6 & 20.3 & -90.00 & 1.40 & 0.52 & 5000 & -2.5 & 4.5 & \\
\hline 73 & $\mathrm{~J} 140012.92+143311.7$ & 210.0538 & 14.5533 & 19.6 & 19.0 & 101.39 & 0.31 & 0.53 & 5000 & -2.0 & 1.5 & $\mathrm{~B}$ \\
\hline 74 & $\mathrm{~J} 140013.33+142618.1$ & 210.0556 & 14.4384 & 18.7 & 18.0 & 44.92 & 0.16 & 0.52 & 4750 & -2.0 & 4.5 & \\
\hline 75 & $\mathrm{~J} 140014.67+143930.7$ & 210.0611 & 14.6585 & 21.3 & 21.0 & 99.91 & 2.29 & 0.55 & 6000 & -1.5 & 4.0 & \\
\hline 76 & $\mathrm{~J} 140015.34+142303.0$ & 210.0640 & 14.3842 & 18.2 & 17.6 & 65.88 & 0.12 & 0.31 & 5250 & -1.0 & 3.5 & \\
\hline 77 & $\mathrm{~J} 140015.57+142348.5$ & 210.0649 & 14.3968 & 18.5 & 17.7 & -53.39 & 0.09 & 0.37 & 4750 & -0.5 & 4.5 & \\
\hline 78 & $\mathrm{~J} 140015.81+143446.8$ & 210.0659 & 14.5797 & 21.7 & 21.4 & 117.33 & 6.40 & 0.84 & 6000 & -2.5 & 4.5 & \\
\hline 79 & J140016.15+143146.9 & 210.0673 & 14.5297 & 21.2 & 20.7 & $\mathrm{X}$ & $\mathrm{X}$ & $\mathrm{X}$ & $\mathrm{X}$ & $\mathrm{X}$ & $\mathrm{X}$ & \\
\hline 80 & $\mathrm{~J} 140016.59+143530.0$ & 210.0691 & 14.5917 & 19.6 & 19.7 & 99.39 & 1.21 & 0.64 & 7500 & -1.0 & 3.0 & \\
\hline 81 & $\mathrm{~J} 140016.62+142925.4$ & 210.0693 & 14.4904 & 21.3 & 20.9 & 97.21 & 2.09 & 0.55 & 5000 & -2.5 & 3.5 & \\
\hline 82 & $\mathrm{~J} 140020.58+143734.0$ & 210.0858 & 14.6261 & 22.1 & 21.7 & 102.01 & 5.57 & 0.90 & 5000 & -2.5 & 1.5 & \\
\hline 83 & $\mathrm{~J} 140021.00+143923.2$ & 210.0875 & 14.6565 & 19.5 & 18.9 & -30.74 & 0.32 & 0.61 & 4750 & -1.0 & 1.5 & \\
\hline 84 & $\mathrm{~J} 140021.84+142553.3$ & 210.0910 & 14.4315 & 21.0 & 20.5 & 90.41 & 1.38 & 0.59 & 5000 & -2.5 & 1.5 & $\mathrm{~B}$ \\
\hline 85 & $\mathrm{~J} 140022.10+143838.2$ & 210.0921 & 14.6439 & 19.5 & 19.7 & 93.84 & 1.52 & 0.55 & 8000 & -1.5 & 3.5 & \\
\hline 86 & $\mathrm{~J} 140022.44+143326.9$ & 210.0935 & 14.5575 & 19.6 & 19.2 & 98.47 & 0.44 & 0.49 & 5250 & -2.0 & 1.5 & B \\
\hline 87 & $\mathrm{~J} 140023.33+142607.9$ & 210.0972 & 14.4356 & 21.9 & 21.3 & 92.79 & 2.30 & 0.56 & 5000 & -1.5 & 1.0 & \\
\hline 88 & $\mathrm{~J} 140023.38+143245.2$ & 210.0974 & 14.5459 & 21.7 & 21.1 & 100.02 & 4.23 & 0.84 & 5000 & -2.5 & 3.0 & \\
\hline 89 & $\mathrm{~J} 140025.16+143346.7$ & 210.1048 & 14.5630 & 21.0 & 20.6 & 100.22 & 1.47 & 0.57 & 5000 & -2.5 & 2.0 & B \\
\hline 90 & $\mathrm{~J} 140025.49+142917.2$ & 210.1062 & 14.4881 & 21.0 & 20.6 & 102.16 & 1.23 & 0.53 & 5000 & -2.5 & 1.0 & B \\
\hline 91 & $\mathrm{~J} 140026.25+143434.4$ & 210.1094 & 14.5762 & 18.8 & 18.2 & 29.74 & 0.17 & 0.50 & 5000 & -1.0 & 4.5 & \\
\hline 92 & $\mathrm{~J} 140026.52+142919.8$ & 210.1105 & 14.4889 & 21.5 & 21.2 & -117.49 & 2.59 & 0.60 & 5000 & -2.0 & 1.5 & \\
\hline 93 & $\mathrm{~J} 140026.57+142948.8$ & 210.1107 & 14.4969 & 21.2 & 20.7 & 108.10 & 1.52 & 0.53 & 5000 & -2.5 & 3.0 & $\mathrm{~B}$ \\
\hline 94 & $\mathrm{~J} 140026.87+144204.2$ & 210.1120 & 14.7012 & 21.9 & 21.5 & -27.44 & 9.14 & 1.00 & 4000 & -2.5 & 3.0 & \\
\hline 95 & $\mathrm{~J} 140027.04+143830.3$ & 210.1127 & 14.6418 & 19.6 & 19.7 & 105.37 & 1.81 & 0.55 & 8000 & -2.0 & 3.0 & B \\
\hline 96 & $\mathrm{~J} 140027.28+143219.5$ & 210.1137 & 14.5388 & 20.3 & 19.8 & 105.68 & 0.79 & 0.51 & 4750 & -2.5 & 1.0 & B \\
\hline 97 & $\mathrm{~J} 140028.13+143311.8$ & 210.1173 & 14.5533 & 22.2 & 22.0 & $\mathrm{X}$ & $\mathrm{X}$ & $\mathrm{X}$ & $\mathrm{X}$ & $\mathrm{X}$ & $\mathrm{X}$ & \\
\hline 98 & $\mathrm{~J} 140028.39+142352.6$ & 210.1183 & 14.3979 & 21.4 & 21.1 & 98.85 & 2.94 & 0.65 & 5000 & -2.5 & 2.0 & \\
\hline 99 & $\mathrm{~J} 140028.73+143142.7$ & 210.1197 & 14.5285 & 21.8 & 21.3 & 2.25 & 2.25 & 0.55 & 5000 & -1.5 & 4.5 & \\
\hline 100 & $\mathrm{~J} 140028.93+142502.2$ & 210.1206 & 14.4173 & 21.8 & 21.4 & 103.40 & 3.65 & 0.58 & 5000 & -2.5 & 2.0 & \\
\hline 101 & $\mathrm{~J} 140028.95+143833.7$ & 210.1206 & 14.6427 & 22.2 & 21.8 & 100.54 & 5.12 & 0.77 & 5000 & -2.5 & 2.0 & \\
\hline 102 & $\mathrm{~J} 140031.33+143718.6$ & 210.1305 & 14.6219 & 21.9 & 21.5 & -43.94 & 3.64 & 0.60 & 5000 & -2.5 & 2.0 & \\
\hline 103 & $\mathrm{~J} 140031.78+142015.5$ & 210.1324 & 14.3376 & 19.2 & 18.7 & 6.44 & 0.41 & 0.49 & 5500 & -1.0 & 4.5 & \\
\hline 104 & $\mathrm{~J} 140031.91+144108.3$ & 210.1329 & 14.6856 & 22.4 & 22.1 & 136.32 & 6.38 & 0.75 & 4000 & -2.5 & 3.5 & \\
\hline 105 & $\mathrm{~J} 140032.14+143627.6$ & 210.1339 & 14.6077 & 21.9 & 21.4 & 164.05 & 3.63 & 0.56 & 5000 & -2.5 & 3.0 & \\
\hline 106 & $\mathrm{~J} 140032.37+142735.8$ & 210.1349 & 14.4600 & 22.2 & 21.9 & $\mathrm{X}$ & $\mathrm{X}$ & $\mathrm{X}$ & $\mathrm{X}$ & $\mathrm{X}$ & $\mathrm{X}$ & \\
\hline 107 & $\mathrm{~J} 140032.54+142400.6$ & 210.1356 & 14.4002 & 19.1 & 18.5 & -60.56 & 0.19 & 0.76 & 5250 & -0.5 & 4.5 & \\
\hline 108 & $\mathrm{~J} 140033.07+142959.7$ & 210.1378 & 14.4999 & 19.7 & 19.2 & 96.86 & 0.44 & 0.52 & 5000 & -2.5 & 2.0 & $\mathrm{~B}$ \\
\hline 109 & $\mathrm{~J} 140033.75+142514.3$ & 210.1406 & 14.4206 & 18.6 & 17.9 & -40.08 & 0.13 & 0.39 & 4750 & -1.0 & 4.5 & \\
\hline 110 & $\mathrm{~J} 140035.97+142854.5$ & 210.1499 & 14.4818 & 21.6 & 21.1 & -123.72 & 2.82 & 0.56 & 5000 & -2.5 & 4.5 & \\
\hline 111 & $\mathrm{~J} 140037.38+142858.0$ & 210.1558 & 14.4828 & 17.4 & 16.4 & 106.95 & 0.16 & 1.00 & 4500 & -2.0 & 1.0 & \\
\hline 112 & $\mathrm{~J} 140039.56+142827.8$ & 210.1648 & 14.4744 & 19.6 & 19.7 & 95.13 & 3.71 & 0.71 & 8000 & -2.5 & 3.5 & \\
\hline 113 & $\mathrm{~J} 140040.90+143208.1$ & 210.1704 & 14.5356 & 21.9 & 21.7 & 106.83 & 4.28 & 0.56 & 6000 & -2.5 & 4.5 & \\
\hline 114 & $\mathrm{~J} 140047.55+142412.0$ & 210.1981 & 14.4033 & 21.0 & 20.5 & 117.74 & 1.62 & 0.61 & 5000 & -2.5 & 2.5 & B \\
\hline 115 & $\mathrm{~J} 140047.57+142630.2$ & 210.1982 & 14.4417 & 21.3 & 20.9 & 99.13 & 2.14 & 0.57 & 5000 & -2.5 & 1.5 & B \\
\hline 116 & $\mathrm{~J} 140053.19+142705.3$ & 210.2217 & 14.4515 & 16.6 & 16.1 & 42.30 & 0.07 & 0.99 & 4500 & 0.0 & 4.5 & \\
\hline 117 & $\mathrm{~J} 140057.91+142854.1$ & 210.2413 & 14.4817 & 21.7 & 21.2 & 103.03 & 3.83 & 0.57 & 5000 & -2.0 & 2.0 & \\
\hline
\end{tabular}

in Figure 14. The velocity dispersion of the lower dispersion component is then $2.4_{-0.5}^{+0.9} \mathrm{~km} \mathrm{~s}^{-1}$, while the velocity dispersion of the other component is not very well determined, but is around $9 \mathrm{~km} \mathrm{~s}^{-1}$. The fraction of stars belonging to the higherdispersion component according to the MCMC fit is around $30 \%$. The corresponding Gaussian distributions are overlaid on the kinematic data in the bottom panel. The mean velocity from our full sample is $\bar{V}_{\mathrm{VLT}}=101.8 \pm 0.7 \mathrm{~km} \mathrm{~s}^{-1}$. In order to assess the probability that the Boötes I velocity distribution is indeed described by two Gaussians instead of one, we measure the likelihood ratio for these two hypotheses, which gives $-2 \log \left(L_{1} / L_{2}\right)=8.06$. This ratio corresponds to a $\sim 98 \%$ confidence of rejecting the single-Gaussian hypothesis.

Our data also provide direct limits on kinematic gradients in Boötes I. We fit the MCMC modeling of the kinematics allowing for a linear gradient in either R.A. (essentially the 


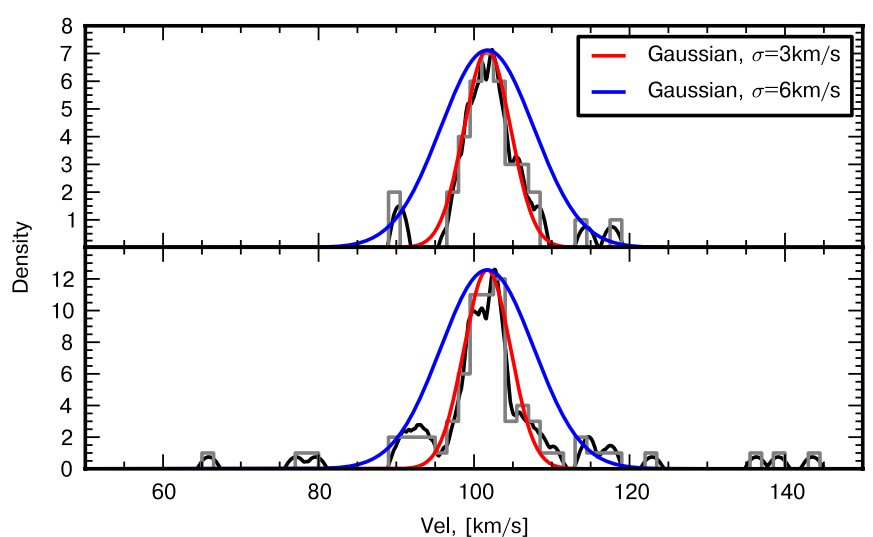

Figure 12. Distribution of stellar velocities in Boötes I. The black line shows the distribution of velocities estimated using the Epanechnikov kernel (Epanechnikov 1969; Wand \& Jones 1995) with a bandwidth of $1.5 \mathrm{~km} \mathrm{~s}^{-1}$, the gray line shows a standard histogram with bin size of $1.5 \mathrm{~km} \mathrm{~s}^{-1}$. The red and blue lines are overplotted Gaussians with sigma of 3 and $6 \mathrm{~km} \mathrm{~s}^{-1}$, respectively; $6 \mathrm{~km} \mathrm{~s}^{-1}$ is the smaller of the previously published determinations of the Boötes I internal velocity dispersion. The top panel shows the velocity distribution for our sample of 37 stars which are highly probable Boötes I members, i.e., those with $[\mathrm{Fe} / \mathrm{H}]<-1.5, \log (g)<3.5$, small velocity error $\sigma_{v}<2.5 \mathrm{~km} \mathrm{~s}^{-1}$, and no significant velocity variability. The bottom panel shows the velocity distribution for all 100 of our stars with non-varying radial velocity.

(A color version of this figure is available in the online journal.)
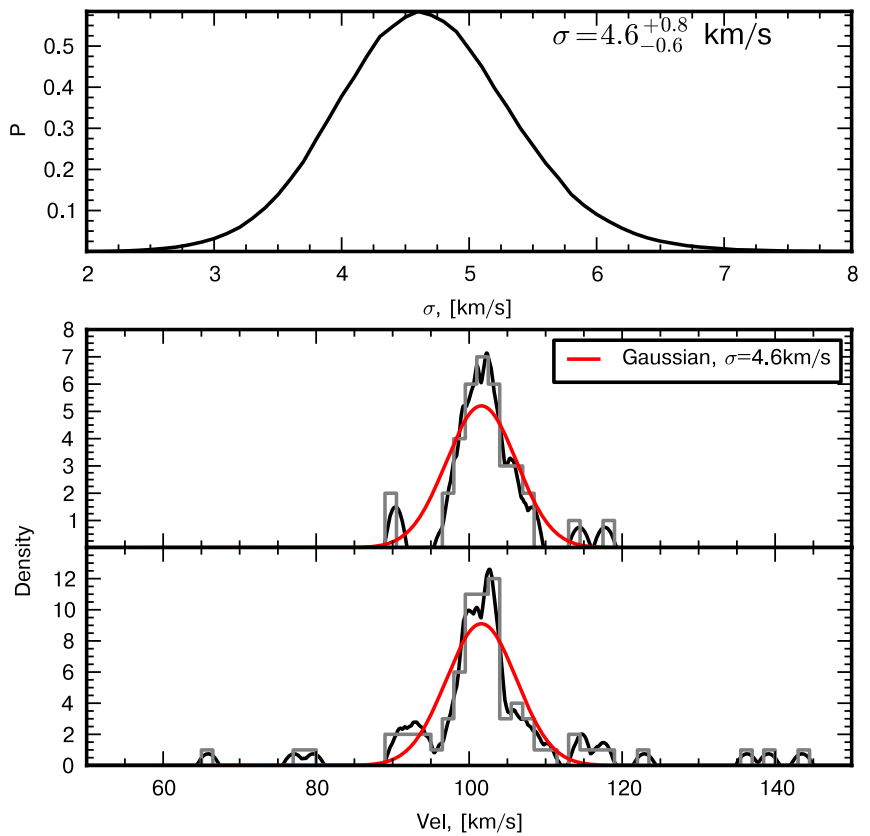

Figure 13. Top panel: the probability distribution of the internal Boötes I Gaussian velocity dispersion, determined from an MCMC fit to our velocity data for our full 100 star non-variable sample, when the velocity distribution is assumed to be consistent with a single Gaussian. Middle panel: the MCMC fit Gaussian, with dispersion $4.6 \mathrm{~km} \mathrm{~s}^{-1}$, overlaid on the kinematic data. Lower panel: to illustrate that the derived MCMC fit is robust to data selection, we show the derived Gaussian with dispersion $4.6 \mathrm{~km} \mathrm{~s}^{-1}$ overlaid on the subset of 37 stars from Figure 12, those which are highly probable Boötes I members, i.e., those with $[\mathrm{Fe} / \mathrm{H}]<-1.5, \log (g)<3.5$, small velocity error $\sigma_{v}<2.5 \mathrm{~km} \mathrm{~s}^{-1}$, and no significant velocity variability.

(A color version of this figure is available in the online journal.)

minor axis) or decl. (essentially the major axis). Our formal limits on rotation are minor axis $-4 \pm 9 \mathrm{~km} \mathrm{~s}^{-1} \mathrm{deg}^{-1}$; major axis $0 \pm 8 \mathrm{~km} \mathrm{~s}^{-1} \mathrm{deg}^{-1}$, recalling that our data cover a radial
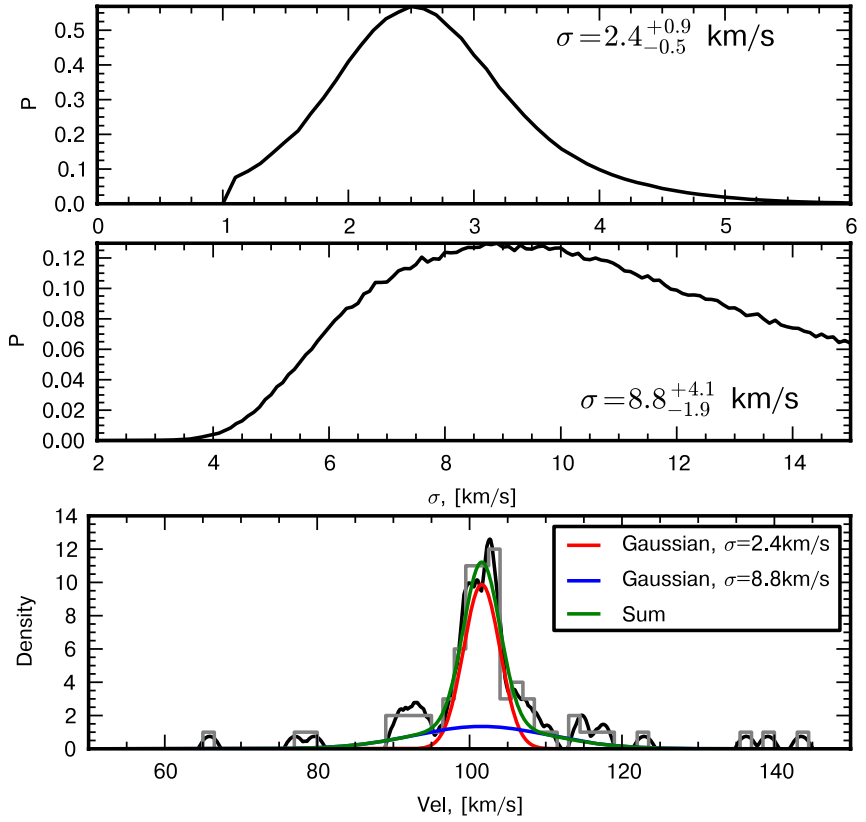

Figure 14. Top two panels: the probability distributions of the internal Boötes I Gaussian velocity dispersion, determined from an MCMC fit to our 100 star nonvariable velocity data, when the velocity distribution is assumed to be consistent with two Gaussians. The top panel shows the probability distribution for the lower dispersion component, while the bottom panel shows the probability distribution for the higher-dispersion component. The MCMC analysis allocated $70 \%$ of the stars to the $2.4 \mathrm{~km} \mathrm{~s}^{-1}$ dispersion component, and $30 \%$ of the stars to the $9 \mathrm{~km} \mathrm{~s}^{-1}$ dispersion component. Lower panel: the corresponding twoGaussian distribution overlaid on the kinematic data.

(A color version of this figure is available in the online journal.)

range of $0.2 \mathrm{deg}$. We may also limit any radial change in the dispersion. This is more difficult, since the minority higherdispersion component becomes dominated by sampling noise if the sample is subdivided by radius. Hence, we fit a singleGaussian velocity dispersion model to the inner half (stars within $0.06 \mathrm{deg}$ of the center of Boötes I) and the outer half of the sample. The difference in derived single-Gaussian dispersion is then (outer minus inner) $=-2.1 \pm 1.3 \mathrm{~km} \mathrm{~s}^{-1}$. This is not a significant result, but hints that the dispersion may be decreasing with radius. That is, the higher-velocity dispersion component may (not significantly) be somewhat more centrally concentrated than is the lower velocity dispersion component.

\section{DISCUSSION AND CONCLUSIONS}

In this paper, we explain how we have developed and implemented a thorough analysis of low-moderate spectral resolution $(R=6500)$ VLT/FLAMES stellar spectra taken in the CaT wavelength region near $860 \mathrm{~nm}$. We optimized a data reduction methodology which delivers very accurate radial velocities and very reliable uncertainties on those radial velocities. We set up an optimized observational proof of methodology, targeting faint candidate RGB stars in the very metal-poor Boötes I dSph galaxy. By making 16 individual repeat observations over six weeks, with a wide dynamic range in each observational data set, we have reached several goals: most importantly, we have been able to properly assess the errors of our radial velocity measurements; our delivered radial velocity precision, for faint extremely weak-lined stars, is better than $1 \mathrm{~km} \mathrm{~s}^{-1}$ for the combined exposures. Second, we have been able to identify and reject stars that show significant radial velocity variability. 
Comparing our derived velocity dispersion and individual velocities with literature studies (Muñoz et al. 2006; Martin et al. 2007), shows that previous studies have substantially overestimated the velocity dispersion of Boötes I. It is possible that earlier studies underestimate their velocity errors, and hence overestimate the velocity distribution which is deconvolved from those errors. Stable fiber-fed spectrographs, including especially VLT+FLAMES, when complemented with an appropriate observational strategy and suitably sophisticated data processing, are able to deliver precise, reliable, and accurate radial velocities with sufficient precision to resolve the intrinsically very low velocity dispersions evident in the low-luminosity dSph satellite galaxies. By exploiting the spectrograph stability to build integrations on times from days to years, we can detect many radial velocity variables, whose unrecognized presence would erroneously inflate a derived velocity dispersion. Importantly, from repeated observations, we can quantify reliably our velocity accuracy. We are currently applying this observational technique to several other $\mathrm{dSph}$ galaxies.

We have useful radial velocity measurements for 100 nonvariable RGB candidate stars, all within one half-light radius (12.5 arcmin, $240 \mathrm{pc}$ ) of the center of Boötes I. Approximately 60-70 stars are likely members from our full sample. Implementing a general MCMC analysis, which includes separate Boötes I member and background distribution functions of our derived stellar parameters $\log (g),[\mathrm{Fe} / \mathrm{H}]$, and of the member and background velocity distributions, we show that the distribution function of stellar radial velocities in Boötes I which we measure can be described in two ways. The less likely is that the distribution is Gaussian, with a velocity dispersion of $4.6_{-0.6}^{+0.8} \mathrm{~km} \mathrm{~s}^{-1}$. The more likely is that the distribution consists of two components: a "colder" component, containing $70 \%$ of the member stars, which has a projected radial velocity dispersion of $2.4_{-0.5}^{+0.9} \mathrm{~km} \mathrm{~s}^{-1}$, and a "hotter" component, containing $30 \%$ of the member stars, which has a projected radial velocity dispersion of $9 \mathrm{~km} \mathrm{~s}^{-1}$. The data favor, with $98 \%$ confidence, the two-component model.

Our data also provide direct limits on kinematic gradients in Boötes I. Our formal limits on rotation are minor axis $-4 \pm 9 \mathrm{~km} \mathrm{~s}^{-1} \mathrm{deg}^{-1}$; major axis $0 \pm 8 \mathrm{~km} \mathrm{~s}^{-1} \mathrm{deg}^{-1}$. Similarly, we may limit any radial change in dispersion. This is more complex, as fitting the full two component with a radial term becomes sample-size-limited. However, fitting a singleGaussian dispersion to the inner half-radius and outer half-radius provides a formal, statistically insignificant, radial decrease in dispersion of $2.1 \pm 1.3 \mathrm{~km} \mathrm{~s}^{-1}$. That is, there is a non-significant hint that the apparently higher-dispersion component is more centrally concentrated than is the whole sample.

We consider now the possibility that the higher-velocity dispersion component in Boötes I kinematics is an artifact of unresolved binaries with variable radial velocities. This paper is based on the data taken during a period of one month. While it definitely allows us to reject stars with significantly variable radial velocities on hour to week timescales, we do not of course identify all binaries. A hypothetical substantial population of binaries with velocity amplitudes of order $10 \mathrm{~km} \mathrm{~s}^{-1}$, and periods much longer than a month, might produce spurious wings in the radial velocity distribution which we would identify as a hotter component. Figure 11 limits the plausibility of this speculation: Martin et al. (2007) observed Boötes I in 2006, we observed Boötes I in 2009-comparison of their velocity data with ours for 27 stars in common identifies perhaps three stars with velocity near that of Boötes I, and with sufficient velocity variability to populate the distribution function wings. This is inconsistent with the results of our MCMC analysis, namely, that some $30 \%$ of stars populate the apparently higher-velocity dispersion component. Thus, taking the data of Martin et al. (2007) at the face value, binary variability is unlikely to be the cause of the higher-dispersion component we detect in our sample.

What can we deduce about the mass of Boötes I? There has been recently a flurry of studies on the means to determine some useful mass parameter to represent a dSph galaxy, where limited radial velocity data are available, concentrated in the central regions. We noted above that the most robust mass that can be estimated for faint dwarfs is the mass enclosed within the half-light radius (for example, among very many studies; Walker et al. 2009; Wolf et al. 2010). These several methods in essence determine $M_{1 / 2}=\beta R_{1 / 2} \sigma_{v}^{2}$, where $M_{1 / 2}$ is a characteristic mass inside the galaxy's half-light radius $R_{1 / 2}, \sigma_{v}^{2}$ is the (Gaussian) dispersion of the line-of-sight radial velocities, and $\beta$ is a factor of value $2.5 / G$ to $3 / G$, with $G$ the Newtonian constant. For a given object, clearly this mass scales as the square of the velocity dispersion. Equally clearly, it assumes that there is a single well-defined Gaussian dispersion.

For Boötes I, we seem to have two dispersions, with no robust way to associate a scale length with either. If we just assume that the dominant low dispersion component is associated with the measured half-light radius, then we can (very approximately) deduce an associated mass within that radius of $240 \mathrm{pc}$. It is perhaps more useful to consider the range of determinations of the half-light mass of Boötes I. Wolf et al. (2010) adopt a velocity dispersion for Boötes I of $9.0 \mathrm{~km} \mathrm{~s}^{-1}$, which they correspond to a mass $M_{1 / 2}=2.36 \times 10^{7} M_{\odot}$ and $M / L=1700$. Fixing the geometric parameters, but adopting the dispersion derived by Martin et al. (2007), $\sigma_{v}=6.5 \mathrm{~km} \mathrm{~s}^{-1}$ corresponds to a mass lower by a factor of 1.9. Adopting our low dispersion of $\sigma_{v}=2.4 \mathrm{~km} \mathrm{~s}^{-1}$ provides a mass lower than that of Wolf et al. (2010) by a factor of 14 , and a corresponding $M / L=120$. The range of these numbers, depending entirely on the quality of the kinematic data, clearly illustrates both the need for excellent quality data to allow useful study of the faint dSphs, and the considerable caution which should be applied to extant analyses of the masses of very low luminosity dSph galaxies.

\subsection{Two Populations in Boötes I: Some Speculation}

Our data suggests that the Boötes I dSph has both cold and hot stellar components. Although the data do not completely rule out a single stellar component with velocity dispersion of $4.6 \mathrm{~km} \mathrm{~s}^{-1}$, that scenario is not favored by our data. In order to understand the origin of the two components we checked whether the components differ in radial distribution or metallicity, but we were not able to find any significant differences. This is not a strong constraint, given (1) that our data extend over only one half-light radius from the center of Boötes I, and (2) our metallicities are unable to resolve the extremely low abundances of Boötes I members. Norris et al. (2010a) show (their Figure 17) that the mean abundance of Boötes $\mathrm{I}$ is $[\mathrm{Fe} / \mathrm{H}]=-2.5$, with a tail down below $[\mathrm{Fe} / \mathrm{H}]=-3.5$. These abundances are below the bottom range of our template spectra and beyond our ability to resolve in this study. At face value, two velocity components correspond to two scale lengths. Might Boötes I have an extended higher-dispersion component? The available Subaru photometry (Okamoto 2010) shows no indication of an extended "envelope" structure, but is not a strong constraint beyond two half-light 
radii, as the stellar surface density is extremely low. We did note in Section 7 that radial velocities are available for two extremely metal-poor members of Boötes I, at distances of 2.0 and 3.9 half-light radii from the center. Both velocities are within $0.5 \mathrm{~km} \mathrm{~s}^{-1}$ of the systemic velocity. While only for two stars, they are consistent with an extremely extended, very metal-poor, low-velocity dispersion component in Boötes I. These two stars were, however, selected for detailed analysis because they were suspected of being very metal-poor. One may not deduce that the more metal-poor stars in Boötes I are the more extended. These two stars do, however, provide some evidence against significant rotation or tidal warping of Boötes I, even out to $1 \mathrm{kpc}$.

Perhaps more interesting, and consistent with a velocity distribution function that is not a single component, is the radial distribution of the 16 member stars with $[\mathrm{Fe} / \mathrm{H}]$ abundances derived by, and listed in Table 3 of, Norris et al. (2010a). If we divide the 16 stars into equal inner and outer groups of eight stars based on radius from the center of Boötes I, the inner and outer groups have mean abundances $[\mathrm{Fe} / \mathrm{H}]=-2.30 \pm 0.12$ and $[\mathrm{Fe} / \mathrm{H}]=-2.78 \pm 0.17$, respectively. These differ at the $2.4 \sigma$ level.

An alternative interpretation is to question the validity of fitting Gaussians to the radial velocity distribution function. In simple models (Gerhard 1993) strong deviations from a Gaussian distribution of radial velocities may arise from the velocity anisotropy of the stellar population. Given our lack of understanding of how $\mathrm{dSph}$ galaxies form, there is no physical basis for assuming a single isotropic velocity distribution. There are two good reasons to doubt the single Gaussian assumption for the very faintest dSph. First, just how does one populate an extremely low-density distribution with scale length $250 \mathrm{pc}$, out to apparently four scale lengths, $1000 \mathrm{pc}$, with a velocity dispersion below $4 \mathrm{~km} \mathrm{~s}^{-1}$ ? Dispersing a central star formation region in a very shallow (cored?) dark matter potential may be feasible. Or it may not. Such a process will inevitably generate very radially biased orbits. One alternative speculation is to merge several star-forming regions, none of which need have been centrally located, during first formation of the dark matter potential which we call Boötes I. This might well generate highly tangentially biased velocity distributions, where one might anticipate the more tangentially biased orbits to be more centrally concentrated. Both processes might happen, so that the velocity distribution may well be bi- or multi-modal in anisotropy, with significant radial gradients in this anisotropy. The least likely expectation is that the velocity distribution function is isotropic-there is no obvious physical process that could generate such a distribution function at such low densities and such large scales.

A plausibility argument of relevance to this speculation is that the lowest luminosity dSphs are systematically less round than are more luminous galaxies_-although Boötes I itself has ellipticity 0.2 , so is relatively round. This flatness may be generated by an isotropic kinematic distribution in a flattened potential, or equally by an anisotropic kinematic distribution in a spherical potential. It is often assumed that $\mathrm{dSph}$ shapes correspond to the shapes of the dominant dark matter distributions. Since one of the very few things we suspect we know about the low-luminosity dSph is that mass does not follow light (more correctly, light does not trace mass, kinematics do), the shape of the luminosity distribution may reflect kinematic anisotropy, not mass anisotropy. In that case radially anisotropic distributions will appear in projected radial velocities as a distribution which is more "cuspy" than is a Gaussian, while tangential anisotropy will appear more extended than is a Gaussian (Gerhard 1993; Binney \& Tremaine 2008). The combination of these effects can look rather like our measured Boötes I velocity distribution in Figure 14.

Thus, one may interpret our statistically preferred twoGaussian radial velocity distribution function in two ways. One option is that there is a two-component structure in Boötes I, with future observations at large Boötes-centric distances required to identify the characteristics of the higher-velocity dispersion population, which is a minority in the inner regions, and which must have a hugely extended radial scale length. Alternatively, Boötes I has a velocity distribution function that reflects its formation, and that is a combination of a majority population with very radially biased orbits, and a minority population with very tangentially biased orbits. Both are represented by a single, measured, radial scale length. The more radially biased population contains the most metal-poor stars.

If this speculation is valid, one anticipates that future precision determination of the velocity distribution function in the most flattened dSph galaxies (Hercules, $e=0.6$ and UMa II $e=$ 0.54 ) will find that neither is consistent with being a single component Gaussian and will find a radially variable shape for the distribution function of radial velocities.

The most important point, which is our conclusion, it is evident that radial velocity data of very high precision, and of extremely well-defined accuracy, are necessary to make progress in defining the kinematics and masses of the faintest dSph galaxies.

We thank Michael Siegel and Ricardo Muñoz for providing us with their photometric variability and kinematic data for Boötes I. Most of the data processing has been done using the python programming language and the following open source modules: numpy, ${ }^{11}$ scipy, ${ }^{12}$ matplotlib, ${ }^{13}$ pypar, ${ }^{14}$ ipython ${ }^{15}$ (Perez \& Granger 2007), astrolibpy, ${ }^{16}$ and pyfits ${ }^{17}$ (pyfits is a product of the Space Telescope Science Institute, which is operated by AURA for NASA). This research has also made use of NASA's Astrophysics Data System Bibliographic Services and TOPCAT software ${ }^{18}$ (Taylor 2005). W.G., D.G., and M.F. gratefully acknowledge financial support for this work from the Chilean Center for Astrophysics FONDAP 15010003, and from the BASAL Center for Astrophysics and Associated Technologies CATA under grant PFB-06/2007. M.G.W. is supported by NASA through Hubble Fellowship grant HST-HF-51283, awarded by the Space Telescope Science Institute, which is operated by the Association of Universities for Research in Astronomy, Inc., for NASA, under contract NAS 5-26555. The data in this study were obtained in ESO Program 182.B-0372A (PI; G. Gilmore).

Facilities: VLT:Kueyen

\section{REFERENCES}

Bailyn, J., \& Ford, A. 2007, MNRAS, 375, L41

Barkana, R., \& Loeb, A. 1999, ApJ, 523, 54

\footnotetext{
11 http://numpy.scipy.org

12 http://www.scipy.org

13 http://matplotlib.sf.net

14 http://code.google.com/p/pypar/

$15 \mathrm{http}: / /$ ipython.scipy.org

$16 \mathrm{http} / / /$ code.google.com/p/astrolibpy/

17 http://www.stsci.edu/resources/software_hardware/pyfits

18 http://www.starlink.ac.uk/topcat/
} 
Baumgardt, H., Côté, P., Hilker, M., Rejkuba, M., Mieske, S., Djorgovski, S. G., \& Stetson, P. 2009, MNRAS, 396, 2051

Belokurov, V., et al. 2006, ApJ, 647, L111

Belokurov, V., et al. 2007, ApJ, 654, 897

Belokurov, V., et al. 2008, ApJ, 686, L83

Belokurov, V., et al. 2009, MNRAS, 397, 1748

Benson, A. J., Bower, R. G., Frenk, C. S., Lacey, C. G., Baugh, C. M., \& Cole, S. 2003, ApJ, 599, 38

Binney, J., \& Tremaine, S. 2008, in Galactic Dynamics, ed. J. Binney \& S. Tremaine (2nd ed.; Princeton, NJ: Princeton Univ. Press), 363

Cappellari, M., \& Emsellem, E. 2004, PASP, 116, 138

Chilingarian, I., Prugniel, P., Sil'Chenko, O., \& Koleva, M. 2007, in IAU Symp. 241, Stellar Populations as Building Blocks of Galaxies, ed. A. Vazdekis \& R. F. Peletie (Cambridge: Cambridge Univ. Press), 175

Clem, J. L., Vanden Berg, D. A., \& Stetson, P. B. 2008, AJ, 135, 682

Cooper, A. P., et al. 2010, MNRAS, 406, 744

Dall'Ora, M., et al. 2006, ApJ, 653, L109

de Jong, J. T. A., Rix, H.-W., Martin, N. F., Zucker, D. B., Dolphin, A. E., Bell, E. F., Belokurov, V., \& Evans, N. W. 2008, AJ, 135, 1361

Epanechnikov, V. A. 1969, Probab. Appl., 14, 153

Geha, M., Willman, B., Simon, J. D., Strigari, L. E., Kirby, E. N., Law, D. R., \& Strader, J. 2009, ApJ, 692, 1464

Gerhard, O. E. 1993, MNRAS, 265, 213

Gilmore, G., Wilkinson, M. I., Wyse, R. F. G., Kleyna, J. T., Koch, A., Evans, N. W., \& Grebel, E. K. 2007, ApJ, 663, 948

Griest, K., Whitmore, J. B., Wolfe, A. M., Prochaska, J. X., Howk, J. C., \& Marcy, G. W. 2010, ApJ, 708, 158

Hanuschik, R. W. 2003, A\&A, 407, 1157

Hargreaves, J. C., Gilmore, G., Irwin, M. J., \& Carter, D. 1994, MNRAS, 269, 957

Hargreaves, J. C., Gilmore, G., \& Annan, J. D. 1996, MNRAS, 279, 108

Hastings, W. K. 1970, Biometrika, 57, 97

Hughes, J., Wallerstein, G., \& Bossi, A. 2008, AJ, 136, 2321

Ibata, R. A., Gilmore, G., \& Irwin, M. J. 1994, Nature, 370, 194

Ibata, R. A., Gilmore, G., \& Irwin, M. J. 1995, MNRAS, 277, 781

Jeffreys, H. 1961, Theory of Probability (3rd ed.; Oxford, UK: Oxford Univ. Press)

Kass, R., \& Raftery, A. 1995, J. Am. Stat. Assoc., 90, 773

Kirby, E. N., Guhathakurta, P., Bolte, M., Sneden, C., \& Geha, M. 2009, ApJ, 705,328

Kirby, E. N., Guhathakurta, P., \& Sneden, C. 2008, ApJ, 682, 1217

Kleyna, J., Wilkinson, M. I., Evans, N. W., Gilmore, G., \& Frayn, C. 2002, MNRAS, 330, 792

Klypin, A., Kravtsov, A. V., Valenzuela, O., \& Prada, F. 1999, ApJ, 522, 82

Koch, A., Wilkinson, M. I., Kleyna, J. T., Gilmore, G. F., Grebel, E. K., Mackey, A. D., Evans, N. W., \& Wyse, R. F. G. 2007, ApJ, 657, 241

Koch, A., et al. 2009, ApJ, 690, 453

Koleva, M., Prugniel, P., Bouchard, A., \& Wu, Y. 2009, A\&A, 501, 1269

Koposov, S., et al. 2008, ApJ, 686, 279

Koposov, S. E., Yoo, J., Rix, H.-W., Weinberg, D. H., Macciò, A. V., \& Escudé, J. M. 2009, ApJ, 696, 2179

Leaman, R., Cole, A. A., Venn, K. A., Tolstoy, E., Irwin, M. J., Szeifert, T., Skillman, E. D., \& McConnachie, A. W. 2009, ApJ, 699, 1

Macciò, A. V., Kang, X., Fontanot, F., Somerville, R. S., Koposov, S., \& Monaco, P. 2010, MNRAS, 402, 1995

Martin, N. F., Ibata, R. A., Chapman, S. C., Irwin, M., \& Lewis, G. F. 2007, MNRAS, 380, 281

Martin, N. F., de Jong, J. T. A., \& Rix, H.-W. 2008, ApJ, 684, 1075

Mateo, M. L. 1998, ARA\&A, 36, 435
McConnachie, A. W., \& Côte, P. 2010, ApJ, 722, L209

Metropolis, N., Rosenbluth, A. W., Rosenbluth, M. N., Teller, A. H., \& Teller, E. 1953, J. Chem. Phys., 21, 1087

Minor, Q. E., Martinez, G., Bullock, J., Kaplinghat, M., \& Trainor, R. 2010, ApJ, 721,1142

Moore, B., Ghigna, S., Governato, F., Lake, G., Quinn, T., Stadel, J., \& Tozzi, P. 1999, ApJ, 524, L19

Munari, U., Sordo, R., Castelli, F., \& Zwitter, T. 2005, A\&A, 442, 1127

Muñoz, R. R., Carlin, J. L., Frinchaboy, P. M., Nidever, D. L., Majewski, S. R., \& Patterson, R. J. 2006, ApJ, 650, L51

Murphy, M. T., Webb, J. K., \& Flambaum, V. V. 2008, MNRAS, 384, 1053

Neal, R. M. 1993, Probabilistic Inference Using Markov Chain Monte Carlo Methods, Technical Report CRG-TR-93-1, (Toronto: Department of Computer Science, University of Toronto)

Norris, J. E., Gilmore, G., Wyse, R. F. G., Wilkinson, M. I., Belokurov, V., Evans, N. W., \& Zucker, D. B. 2008, ApJ, 689, L113

Norris, J. E., Wyse, R. F. G., Gilmore, G., Yong, D., Frebel, A., Wilkinson, M. I., Belokurov, V., \& Zucker, D. B. 2010a, ApJ, 723, 1632

Norris, J. E., Yong, D., Gilmore, G., \& Wyse, R. F. G. 2010b, ApJ, 711, 350

Okamoto, S. 2010, PhD thesis, Tokyo Univ.

Olszewski, E. W., Pryor, C., \& Armandroff, T. E. 1996, AJ, 111, 750

Pasquini, L., et al. 2002, Messenger, 110, 1

Patil, A., Huard, D., \& Fonnesbeck, C. J. 2010, J. Stat. Softw., 35, 1

Penarrubia, J., McConnachie, A. W., \& Navarro, J. F. 2008, ApJ, 672, 904

Perez, F., \& Granger, B. E. 2007, Comput. Sci. Eng., 9, 21

Prugniel, P., Soubiran, C., Koleva, M., \& Le Borgne, D. 2007, arXiv:astro-ph/ 0703658

Ricotti, M. 2010, Adv. Astron., 2010, 271592

Rix, H.-W., \& White, S. D. M. 1992, MNRAS, 254, 389

Rousseeuw, P. J., \& Croux, C. 1993, J. Am. Stat. Assoc., 88, 1273

Schlegel, D. J., Finkbeiner, D. P., \& Davis, M. 1998, ApJ, 500, 525

Siegel, M. H. 2006, ApJ, 649, L83

Simkin, S. M. 1974, A\&A, 31, 129

Simon, J. D., \& Geha, M. 2007, ApJ, 670, 313

Somerville, R. S. 2002, ApJ, 572, L23

Taylor, M. B. 2005, in ASP Conf. Ser. 347, Astronomical Data Analysis Software and Systems XIV, ed. P. Shopbell, M. Britton, \& R. Ebert (San Francisco, CA: ASP), 29

Tollerud, E. J., Bullock, J. S., Strigari, L. E., \& Willman, B. 2008, ApJ, 688, 277

Tolstoy, E., Hill, V., \& Tosi, M. 2009, ARA\&A, 47, 371

Tonry, J., \& Davis, M. 1979, AJ, 84, 1511

Trotta, R. 2007, MNRAS, 378, 72

Walker, M. G., Mateo, M., Olszewski, E. W., Gnedin, O. Y., Wang, X., Sen, B., \& Woodroofe, M. 2007, ApJ, 667, L53

Walker, M. G., Mateo, M., Olszewski, E. W., Pearrubia, J., Wyn Evans, N., \& Gilmore, G. 2009, ApJ, 704, 1274

Walsh, S. M., Jerjen, H., \& Willman, B. 2007, ApJ, 662, L83

Walsh, S. M., Willman, B., \& Jerjen, H. 2009, AJ, 137, 450

Wand, M. P., \& Jones, M. C. 1995, Kernel Smoothing (London: Chapman and Hall)

Wilkinson, M. I., Kleyna, J., Evans, N. W., \& Gilmore, G. 2002, MNRAS, 330, 778

Willman, B., et al. 2005, ApJ, 626, L85

Wolf, J., Martinez, G. D., Bullock, J. S., Kaplinghat, M., Geha, M., Munoz, R. R., Simon, J. D., \& Avedo, F. F. 2010, MNRAS, 406, 1220

York, D. G., et al. 2000, AJ, 120, 1579

Zucker, D. B., et al. 2006a, ApJ, 643, L103

Zucker, D. B., et al. 2006b, ApJ, 650, L41 